\title{
CATEGORIES OF HOLOMORPHIC VECTOR BUNDLES ON NONCOMMUTATIVE TWO-TORI
}

\author{
A. POLISHCHUK AND A. SCHWARZ
}

\begin{abstract}
In this paper we study the category of standard holomorphic vector bundles on a noncommutative two-torus. We construct a functor from the derived category of such bundles to the derived category of coherent sheaves on an elliptic curve and prove that it induces an equivalence with the subcategory of stable objects. By the homological mirror symmetry for elliptic curves this implies an equivalence between the derived category of holomorphic bundles on a noncommutative two-torus and the Fukaya category of the corresponding symplectic (commutative) torus.
\end{abstract}

\section{INTRODUCTION}

In this paper we study holomorphic vector bundles on noncommutative 2-dimensional torus $T_{\theta}$, where $\theta \in \mathbb{R}$, following the approach of [19], [16], and [3]. More precisely, we equip $T_{\theta}$ with a complex structure given by some $\tau \in \mathbb{C} \backslash \mathbb{R}$ and study analogues of $\bar{\partial}$-connections on vector bundles over the complex noncommutative torus $T_{\theta, \tau}$. Recall that vector bundles correspond to projective modules over the algebra $A_{\theta}$ of smooth functions over $T_{\theta}$. As in [3] we restrict our attention to standard holomorphic structures on basic $A_{\theta}$-modules, for which there exist compatible connections of constant curvature. We do not consider here the problem how to characterize such holomorphic bundles although it seems that similar to the commutative case the answer should involve some notion of stability (see remarks at the end of 2.1). Motivated by the calculations of [8] we prove that the derived category of standard holomorphic vector bundles on $T_{\theta, \tau}$ is equivalent to the full subcategory of stable objects in the derived category $D^{b}\left(X_{\tau}\right)$ of coherent sheaves on the complex elliptic curve $X_{\tau}=\mathbb{C} /(\mathbb{Z}+\tau \mathbb{Z})$. In particular, the former category does not depend on $\theta$. Under this equivalence standard holomorphic bundles on $T_{\theta, \tau}$ land into an abelian subcategory of $D^{b}\left(X_{\tau}\right)$ obtained by certain tilting of the category $\operatorname{Coh}\left(X_{\tau}\right)$ of coherent sheaves on $X_{\tau}$ depending on $\theta$. Roughly speaking, to get this abelian subcategory of $D^{b}\left(X_{\tau}\right)$, one has to cut $\operatorname{Coh}\left(X_{\tau}\right)$ into two subcategories generated by stable bundles of slopes $<\theta$ and $>\theta$ respectively (we assume that $\theta$ irrational) and then reassemble these subcategories in a different way into a new abelian category. The general framework for such constructions is provided by torsion theory of [7]. These $t$-structures were considered by Bridgeland in connection with general stability conditions for derived categories.

In physical terms the objects of our study are D-branes of B-type in SUSY Yang-Mills theory on noncommutative 2-tori. Although the corresponding derived categories are the same for all $\theta$, the physical branes (stable objects) do depend on $\theta$. This resembles the picture where stability conditions on the derived category of

The work of both authors was partially supported by NSF grants. 
coherent sheaves over a Calabi-Yau manifold vary with Kähler structure. (see [5] for more general discussion).

An interesting feature of the above equivalence of categories is that it is compatible with Morita equivalences and the action of $\mathrm{SL}_{2}(\mathbb{Z})$ on $D^{b}\left(X_{\tau}\right)$ defined by Mukai (see [10]). Namely, for every $g \in \mathrm{SL}_{2}(\mathbb{Z})$ one can consider the fractionallinear action of $g$ on parameter $\theta$. The noncommutative tori $T_{g \theta}$ and $T_{\theta}$ are Morita equivalent, i.e., there exists an $A_{g \theta}-A_{\theta}$-bimodule inducing an equivalence of categories of modules over $A_{g \theta}$ and $A_{\theta}$. This equivalence extends to an equivalence between categories of holomorphic bundles on $T_{g \theta, \tau}$ and $T_{\theta, \tau}$. Now one can compare equivalences between the corresponding derived categories with $D^{b}\left(X_{\tau}\right)$. It turns out that they differ by the action of the autoequivalence of $D^{b}\left(X_{\tau}\right)$ corresponding to $g^{t}$. Thus, our picture can be considered as a "noncommutative explanation" of the existence of the Fourier-Mukai transform.

We consider two approaches to constructing the above equivalences. One uses the explicit calculation of structure constants of products of noncommutative theta functions in [3]. Another is based on a construction of the Fourier-Mukai type functor from the category of holomorphic vector bundles on $T_{\theta, \tau}$ to the category of complexes of sheaves of $\mathcal{O}$-modules with coherent cohomology on $X_{\tau}$.

We restrict ourselves to considering only standard holomorphic structures on basic projective modules over $A_{\theta}$, which corresponds to considering only stable vector bundles on the commutative elliptic curve (or more generally, simple coherent sheaves). However, we believe that the functor we construct extends to all holomorphic bundles on $T_{\theta}$. It is also clear that some of the results of this paper can be generalized to higher-dimensional noncommutative tori. Note that in this generalization sometimes one has to replace the category of coherent sheaves on the corresponding commutative complex torus $X$ by the twisted category associated with some cohomology class in $H^{2}\left(X, \mathcal{O}^{*}\right)$ (this twisting is always trivial in 2-dimensional case).

In the paper [8] that motivated us the category of standard holomorphic bundles on $T_{\theta}$ is compared with the Fukaya category of a symplectic torus. In view of the equivalence we establish in this paper, such a comparison essentially reduces to the usual homological mirror symmetry for an elliptic curve proved in [13]. It is remarkable that the functor from the Fukaya category to the category of holomorphic bundles on $T_{\theta}$ considered in [8] seems to be simplier than the similar functor in the commutative case. It would be interesting to study the relation between the higherdimensional generalization of this functor and Fukaya's work on noncommutative mirror symmetry [6].

We should mention that a different construction of categories associated with noncommutative elliptic curves was proposed by Soibelman and Vologodsky in [18]. The categories $\mathcal{B}_{q}$ they construct depend only on one parameter $q=\exp (2 \pi i \theta)$, while our categories depend on two parameters $\theta$ and $\tau$. It seems plausible that the categories $\mathcal{B}_{q}$ can be obtained as some asymptotic limits of our categories when $\tau$ goes to infinity. On the other hand, the categories $\mathcal{B}_{q}$ are in some sense degenerations of the categories of coherent sheaves on commutative elliptic curves $\mathbb{C}^{*} / q^{\mathbb{Z}}$ (where $|q|<1$ ) as $|q| \rightarrow 1$. The general philosophy of this degeneration procedure was outlined in [17]. 
It would be also interesting to understand the role of holomorphic structures in Manin's program that is supposed to relate the real multiplication for noncommutative two-tori with arithmetics of real quadratic fields (see [9]).

Acknowledgment. We are grateful to Marc Rieffel, Yan Soibelman and Mauro Spera for useful discussions. Part of this research was carried out during the stay of both authors at the Institut des Hautes Etudes Scientifiques. We thank this institution for the hospitality and for stimulating working conditions.

\section{Preliminaries}

1.1. Basic modules. Let $\theta$ be a real number, $T_{\theta}$ be the corresponding 2 -dimensional noncommutative torus. By the definition, the algebra $A_{\theta}$ of smooth functions on $T_{\theta}$ consists of formal linear combinations $\sum_{\left(n_{1}, n_{2}\right) \in \mathbb{Z}^{2}} a_{n_{1}, n_{2}} U_{1}^{n_{1}} U_{2}^{n_{2}}$ with the coefficient function $\left(n_{1}, n_{2}\right) \mapsto a_{n_{1}, n_{2}}$ rapidly decreasing at infinity. The product is defined using the rule

$$
U_{1} U_{2}=\exp (2 \pi i \theta) U_{2} U_{1}
$$

We are interested in finitely generated projective right $A_{\theta}$-modules. It is known (see [1], [14]) that every such module is isomorphic to one of the following modules $E_{n, m}(\theta)$, where $(m, n)$ is a pair of integers such that $n+m \theta \neq 0$. Assume first that $m \neq 0$. Then $E_{n, m}(\theta)$ is defined as the Schwartz space $\mathcal{S}(\mathbb{R} \times \mathbb{Z} / m \mathbb{Z})$ equipped with the following right action of $A_{\theta}$ :

$$
f U_{1}(x, \alpha)=f\left(x-\frac{n+m \theta}{m}, \alpha-1\right), f U_{2}(x, \alpha)=\exp \left(2 \pi i\left(x-\frac{\alpha n}{m}\right)\right) f(x, \alpha),
$$

where $x \in \mathbb{R}, \alpha \in \mathbb{Z} / m \mathbb{Z}$. For $m=0$ and $n \neq 0$ we set $E_{n, 0}(\theta)=A_{\theta}^{|n|}$ with the obvious right $A_{\theta}$-action. Let us denote by

$$
\begin{gathered}
\operatorname{deg}\left(E_{n, m}(\theta)\right)=m, \\
\operatorname{rk}\left(E_{n, m}(\theta)\right)=n+m \theta, \\
\mu\left(E_{n, m}(\theta)\right)=\frac{m}{n+m \theta}
\end{gathered}
$$

the natural analogues of degree, rank and slope. The modules $E_{-n,-m}(\theta)$ and $E_{n, m}(\theta)$ are equivalent, however, sometimes we would like to distinguish them by introducing the $\mathbb{Z} / 2 \mathbb{Z}$-grading. Namely, we will say that the module $E$ is even if $\operatorname{rk} E>0$ and that it is odd if $\mathrm{rk} E<0$. Thus, in the category of $\mathbb{Z} / 2 \mathbb{Z}$-graded projective $A_{\theta}$-modules we have

$$
E_{-n,-m}(\theta)=\Pi E_{n, m}(\theta)
$$

where $E \mapsto \Pi E$ denotes the parity change. In the case when $\theta$ is rational, we could also try to use the above formulas to define modules associated with $(m, n)$ such that $n+m \theta=0$; however, these modules are not projective.

Basic modules are the modules $E_{n, m}(\theta)$ with $m$ and $n$ relatively prime. It is known that for $(m, n)$ relatively prime one has $E_{n d, m d}(\theta) \simeq E_{n, m}(\theta)^{\oplus d}$.

It is often convenient to extend the pair $(m, n)$ to a matrix in $\mathrm{SL}_{2}(\mathbb{Z})$. When we need to make this choice we will use the following notation: for a matrix $g=$ $\left(\begin{array}{ll}a & b \\ c & d\end{array}\right) \in \mathrm{SL}_{2}(\mathbb{Z})$ we set $E_{g}(\theta)=E_{d, c}(\theta)$. In particular, for $g=1$, we get $E_{1}(\theta)=$ $E_{1,0}(\theta)=A_{\theta}$. In accordance with our formulas for the degree and rank of modules we set for a matrix $g$ as above

$$
\operatorname{deg}(g)=c
$$




$$
\operatorname{rk}(g, \theta)=c \theta+d,
$$

so that $\operatorname{deg}\left(E_{g}(\theta)\right)=\operatorname{deg}(g)$ and $\operatorname{rk}\left(E_{g}(\theta)\right)=\operatorname{rk}(g, \theta)$. Note that the function $g \mapsto \operatorname{rk}(g, \theta)$ satisfies the following cocycle condition

$$
\operatorname{rk}\left(g_{1} g_{2}, \theta\right)=\operatorname{rk}\left(g_{1}, g_{2} \theta\right) \operatorname{rk}\left(g_{2}, \theta\right)
$$

where we denote by $\theta \mapsto g \theta=(a \theta+b) /(c \theta+d)$ the natural action of $\mathrm{SL}_{2}(\mathbb{Z})$ on the set of $\theta$ 's (it is always well-defined for irrational $\theta$, and partially defined for rational $\theta)$. We will also need the following identity which can be easily checked:

$$
\operatorname{deg}\left(g_{2} g_{1}^{-1}\right) \operatorname{rk}\left(g_{3}, \theta\right)-\operatorname{deg}\left(g_{3} g_{1}^{-1}\right) \operatorname{rk}\left(g_{2}, \theta\right)+\operatorname{deg}\left(g_{3} g_{2}^{-1}\right) \operatorname{rk}\left(g_{1}, \theta\right)=0 .
$$

It is well-known that endomorphisms of the basic right $A_{\theta}$-module $E_{g}(\theta)$ can be identified with the algebra $A_{g \theta}$. The corresponding left action of $A_{g \theta}$ on $E_{g}(\theta)$ is given by the formulas

$$
U_{1} f(x, \alpha)=f\left(x-\frac{1}{c}, \alpha-a\right), U_{2} f(x, \alpha)=\exp \left(2 \pi i\left(\frac{x}{c \theta+d}-\frac{\alpha}{c}\right)\right) f(x, \alpha),
$$

where $g=\left(\begin{array}{ll}a & b \\ c & d\end{array}\right)$.

1.2. Pairings. We have a canonical pairing

$$
b: E_{g^{-1}}(g \theta) \otimes_{\mathbb{C}} E_{g}(\theta) \rightarrow \mathbb{C}
$$

defined by the formula

$$
b\left(f_{1} \otimes f_{2}\right)=\sum_{\alpha \in \mathbb{Z} / \operatorname{deg}(g) \mathbb{Z}} \int_{x \in \mathbb{R}} f_{1}\left(\frac{x}{\operatorname{rk}(g, \theta)}, \alpha\right) f_{2}(x,-a \alpha) d x,
$$

where $f_{1} \in E_{g^{-1}}(g \theta), f_{2} \in E_{g}(\theta), g$ is given by the matrix above.

Lemma 1.1. For every $U \in A_{g \theta}, V \in A_{\theta}$ one has

$$
\begin{aligned}
& b\left(f_{1} U \otimes f_{2}\right)=b\left(f_{1} \otimes U f_{2}\right), \\
& b\left(V f_{1} \otimes f_{2}\right)=b\left(f_{1} \otimes f_{2} V\right) .
\end{aligned}
$$

Proof. It suffices to check these equalities when $U$ (resp. $V$ ) is one of the two generators of the algebra $A_{g \theta}$ (resp. $A_{\theta}$ ), in which case it is straighforward.

Proposition 1.2. (a) For every $g_{1}, g_{2} \in \mathrm{SL}_{2}(\mathbb{Z})$ one has a well-defined pairing of right $A_{\theta}$-modules

$$
t_{g_{1}, g_{2}}: E_{g_{1}}\left(g_{2} \theta\right) \otimes_{\mathbb{C}} E_{g_{2}}(\theta) \rightarrow E_{g_{1} g_{2}}(\theta)
$$

given by the following formulas. If $g_{1} \neq 1, g_{2} \neq 1$ and $g_{1} g_{2} \neq 1$ then $t_{g_{1}, g_{2}}\left(f_{1} \otimes f_{2}\right)(x, \alpha)=$

$$
\begin{gathered}
\sum_{n \in \mathbb{Z}} f_{1}\left(\frac{x}{\operatorname{rk}\left(g_{2}, \theta\right)}+\frac{\operatorname{rk}\left(g_{1}, g_{2} \theta\right)}{c_{1}}\left(\frac{c_{2} d_{12} \alpha}{c_{12}}-n\right), a_{1} d_{12} \alpha-n\right) f_{2}\left(x-\frac{d_{12} \alpha}{c_{12}}+\frac{n}{c_{2}}, a_{2} n\right), \\
\text { where } x \in \mathbb{R}, \alpha \in \mathbb{Z} / c_{12} \mathbb{Z}, g_{i}=\left(\begin{array}{ll}
a_{i} & b_{i} \\
c_{i} & d_{i}
\end{array}\right), g_{1} g_{2}=\left(\begin{array}{ll}
a_{12} & b_{12} \\
c_{12} & d_{12}
\end{array}\right) \text {. The pairing } \\
t_{g, 1}: E_{g}(\theta) \otimes_{\mathbb{C}} E_{1}(\theta) \rightarrow E_{g}(\theta)
\end{gathered}
$$

is given by the right action of the algebra $A_{\theta}=E_{1}(\theta)$ on $E_{g}(\theta)$. The pairing

$$
t_{1, g}: E_{1}(g \theta) \otimes_{\mathbb{C}} E_{g}(\theta) \rightarrow E_{g}(\theta)
$$


is set to be the left action of the algebra $A_{g \theta}=E_{1}(g \theta)$ on $E_{g}(\theta)$ defined before. Finally, the pairing

$$
t_{g^{-1}, g}: E_{g^{-1}}(g \theta) \otimes_{\mathbb{C}} E_{g}(\theta) \rightarrow E_{1}(\theta)=A_{\theta}
$$

is given by the formula

$$
t_{g^{-1}, g}\left(f_{1} \otimes f_{2}\right)=\sum_{\left(n_{1}, n_{2}\right) \in \mathbb{Z}^{2}} U_{1}^{n_{1}} U_{2}^{n_{2}} b\left(U_{2}^{-n_{2}} U_{1}^{-n_{1}} f_{1} \otimes f_{2}\right),
$$

where $b$ is the pairing (1.3).

(b) For every triple of elements $g_{1}, g_{2}, g_{3} \in \mathrm{SL}_{2}(\mathbb{Z})$ the following diagram is commutative

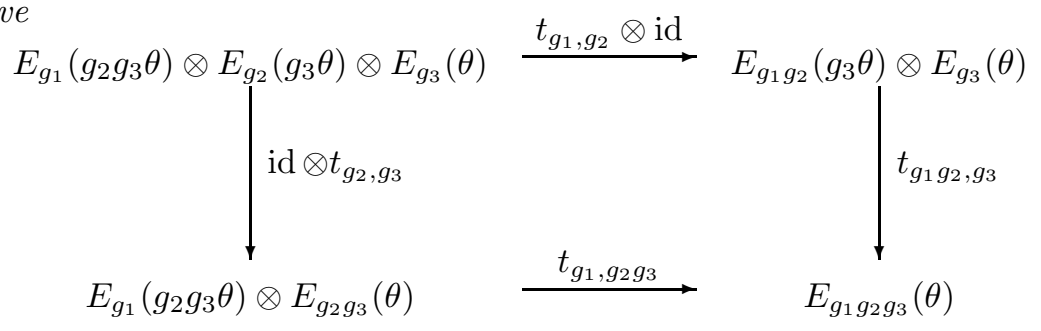

(c) The map $E_{g_{1}}\left(g_{2} \theta\right) \rightarrow \operatorname{Hom}_{A_{\theta}}\left(E_{g_{2}}(\theta), E_{g_{1} g_{2}}(\theta)\right)$ induced by $t_{g_{1}, g_{2}}$ is an isomorphism.

Before starting the proof it is convenient to rewrite the formula for $t_{g_{1}, g_{2}}$ a little bit.

Lemma 1.3. (a) For $g_{1}, g_{2} \in \mathrm{SL}_{2}(\mathbb{Z})$ such that $g_{1} \neq 1, g_{2} \neq 1, g_{1} g_{2} \neq 1$ one has

$$
\begin{aligned}
& t_{g_{1}, g_{2}}\left(f_{1} \otimes f_{2}\right)(x, \alpha)=\sum_{\alpha_{1} \in \mathbb{Z} / c_{1} \mathbb{Z}, \alpha_{2} \in \mathbb{Z} / c_{2} \mathbb{Z}} f_{n \in I_{g_{1}, g_{2}}\left(\alpha_{1}, \alpha_{2}, \alpha\right)} f_{1}\left(\frac{x}{\operatorname{rk}\left(g_{2}, \theta\right)}+\frac{\operatorname{rk}\left(g_{1}, g_{2} \theta\right) n}{c_{1} c_{12}}, \alpha_{1}\right) f_{2}\left(x-\frac{n}{c_{2} c_{12}}, \alpha_{2}\right), \\
& \quad
\end{aligned}
$$

where $g_{1}, g_{2}$ and $g_{1} g_{2}$ are given by the same matrices as in the above proposition,

$$
\begin{aligned}
& I_{g_{1}, g_{2}}\left(\alpha_{1}, \alpha_{2}, \alpha\right)=\left\{n \in \mathbb{Z} \mid n \equiv-c_{1} \alpha+c_{12} \alpha_{1}\left(c_{12} c_{1}\right),\right. \\
& \left.n \equiv c_{2} d_{12} \alpha-c_{12} d_{2} \alpha_{2}\left(c_{12} c_{2}\right)\right\} .
\end{aligned}
$$

(b) For $n \in I_{g_{1}, g_{2}}\left(\alpha_{1}, \alpha_{2}, \alpha\right)$ one has $n \equiv-c_{1} \alpha_{2}+d_{1} c_{2} \alpha_{1}\left(c_{1} c_{2}\right)$.

(c) One has $I_{g_{2},-g_{2}^{-1} g_{1}^{-1}}\left(\alpha_{1}, \alpha_{2}, \alpha_{3}\right)=I_{g_{1}, g_{2}}\left(-a_{1} \alpha_{3},-\alpha_{1},-a_{12} \alpha_{2}\right)$.

Proof of lemma. (a) In the formula of Proposition 1.2(a) one should change the summation variable by setting $n^{\prime}=c_{2} d_{12} \beta-c_{12} n$. Then the congruences

$$
a_{1} d_{12} \alpha-n \equiv \alpha_{1}\left(c_{1}\right), a_{2} n \equiv \alpha_{2}\left(c_{2}\right)
$$

are equivalent to the congruences

$$
n^{\prime} \equiv c_{2} d_{12} \alpha-c_{12} d_{2} \alpha_{2}\left(c_{12} c_{2}\right), n^{\prime} \equiv\left(c_{2}-a_{1} c_{12}\right) d_{12} \alpha+c_{12} \alpha_{1}\left(c_{12} c_{1}\right)
$$

(we used the fact that $\left.d_{2} \equiv a_{2}^{-1}\left(c_{2}\right)\right)$. It remains to use the relation $\left(c_{2}-a_{1} c_{12}\right) d_{12}=$ $-c_{1} a_{12} d_{12} \equiv-c_{1}\left(c_{12} c_{1}\right)$ to see that these congruences are equivalent to $n^{\prime} \in$ $I_{g_{1}, g_{2}}\left(\alpha_{1}, \alpha_{2}, \alpha\right)$.

(b) Let us pick a representative in $\mathbb{Z}$ for $\alpha \in \mathbb{Z} / c_{12} \mathbb{Z}$. Since $c_{2} d_{12}-c_{12} d_{2}=-c_{1}$ we have the congruence

$$
n \equiv-c_{1} \alpha+c_{12} d_{2}\left(\alpha-\alpha_{2}\right)\left(c_{12} c_{2}\right)
$$


for $n \in I_{g_{1}, g_{2}}\left(\alpha_{1}, \alpha_{2}, a\right)$. Hence, $k:=\left(n+c_{1} \alpha\right) / c_{12}$ is an integer and we have

$$
k \equiv d_{2}\left(\alpha-\alpha_{2}\right)\left(c_{2}\right), k \equiv \alpha_{1}\left(c_{1}\right) .
$$

Using these two relations we get

$n+c_{1} \alpha=c_{12} k=c_{1} a_{2} k+d_{1} c_{2} k \equiv c_{1} a_{2} d_{2}\left(\alpha-\alpha_{2}\right)+d_{1} c_{2} \alpha_{1}\left(c_{1} c_{2}\right) \equiv c_{1}\left(\alpha-\alpha_{2}\right)+d_{1} c_{2} \alpha_{1}\left(c_{1} c_{2}\right)$

as required.

(c) This follows easily from (b).

Proof of Proposition 1.2. The pairing $t_{g_{1}, g_{2}}$ for $g_{1} \neq 1, g_{2} \neq 1, g_{1} g_{2} \neq 1$ is essentially the same as in section 2 of [3]. We just replaced left modules by right modules everywhere. Now the results of [3] imply that $t_{g_{1}, g_{2}}$ for $g_{1} \neq 1, g_{2} \neq 1$ gives rise to a map of $A_{g_{1} g_{2} \theta}-A_{\theta}$-bimodules (in fact, an isomorphism)

$$
E_{g_{1}}\left(g_{2} \theta\right) \otimes_{A_{g_{2} \theta}} E_{g_{2}}(\theta) \rightarrow E_{g_{1} g_{2}}(\theta) .
$$

This proves the associativity in (b) for the case when one of the elements $g_{i}$ is equal to 1 , and two other elements $g_{j}$ and $g_{k}$ are different from 1 and are not inverse to each other. It is also easy to see directly that the pairing $t_{g^{-1}, g}$ gives rise to a map of $A_{\theta}$ - $A_{\theta}$-bimodules (also an isomorphism)

$$
E_{g^{-1}}(g \theta) \otimes_{A_{g \theta}} E_{g}(\theta) \rightarrow A_{\theta}
$$

which implies the associativity in (b) for the triples $\left(g^{-1}, g, 1\right),\left(g^{-1}, 1, g\right)$ and $\left(1, g^{-1}, g\right)$.

In the case when all the elements $g_{1}, g_{2}, g_{3}, g_{1} g_{2}, g_{2} g_{3}, g_{1} g_{2} g_{3}$ are non-trivial the associativity can be checked by a straightforward calculation using Lemma 1.3(a),(b). More precisely, if we denote $g_{i}=\left(\begin{array}{ll}a_{i} & b_{i} \\ c_{i} & d_{i}\end{array}\right), g_{i} g_{j}=\left(\begin{array}{ll}a_{i j} & b_{i j} \\ c_{i j} & d_{i j}\end{array}\right), g_{1} g_{2} g_{3}=$ $\left(\begin{array}{ll}a_{123} & b_{123} \\ c_{123} & d_{123}\end{array}\right)$, then the associativity follows from the statement that for every collection $\left(\alpha_{1}, \alpha_{2}, \alpha_{3}, \alpha\right)$, where $\alpha_{i} \in \mathbb{Z} / c_{i} \mathbb{Z}, \alpha \in \mathbb{Z} / c_{123} \mathbb{Z}$, the map $(m, n) \mapsto$ $\left(\left(c_{3} m+c_{123} n\right) / c_{23},\left(c_{2} m-c_{1} n\right) / c_{23}\right)$ gives a bijection

$$
\begin{aligned}
& \cup_{\alpha_{23} \in \mathbb{Z} / c_{23} \mathbb{Z}} I_{g_{1}, g_{2} g_{3}}\left(\alpha_{1}, \alpha_{23}, \alpha\right) \times I_{g_{2}, g_{3}}\left(\alpha_{2}, \alpha_{3}, \alpha_{23}\right) \widetilde{\rightarrow} \\
& \cup_{\alpha_{12} \in \mathbb{Z} / c_{12} \mathbb{Z}} I_{g_{1} g_{2}, g_{3}}\left(\alpha_{12}, \alpha_{3}, \alpha\right) \times I_{g_{1}, g_{2}}\left(\alpha_{1}, \alpha_{2}, \alpha_{12}\right) .
\end{aligned}
$$

The remaining interesting cases are: (i) $g_{1} g_{2} g_{3}=1$, all elements $g_{1}, g_{2}, g_{3}$ are non-trivial; (ii) $g_{1} g_{2}=1$, all elements $g_{2}, g_{3}, g_{2} g_{3}$ are non-trivial; (ii)' $g_{2} g_{3}=1$, all elements $g_{1}, g_{2}, g_{1} g_{2}$ are non-trivial. The case (i) boils down to the identity

$$
b\left(t_{g_{1}, g_{2}}\left(f_{1} \otimes f_{2}\right) \otimes f_{3}\right)=b\left(f_{1}, t_{g_{2}, g_{3}}\left(f_{2} \otimes f_{3}\right)\right)
$$

which is not difficult to check directly. Let us prove the identity in the case (ii) (the case (ii)' is similar). We can assume that $\theta$ is irrational (the case of rational $\theta$ will follow by continuity). Then we can easily show that the associativity holds up to a constant. Indeed, for fixed $f_{1} \in E_{g_{2}^{-1}}\left(g_{2} g_{3} \theta\right), f_{2} \in E_{g_{2}}\left(g_{3} \theta\right)$, the map

$$
f_{3} \mapsto t_{g_{2}^{-1}, g_{2} g_{3}}\left(f_{1} \otimes t_{g_{2}, g_{3}}\left(f_{2} \otimes f_{3}\right)\right.
$$

is a morphism of right $A_{\theta}$-modules $E_{g_{3}}(\theta) \rightarrow E_{g_{3}}(\theta)$, hence it is given by the left action of some element $t^{\prime}\left(f_{1}, f_{2}\right) \in A_{g_{3} \theta}$. Moreover, the map $t^{\prime}$ gives a morphism of $A_{g_{3} \theta}-A_{g_{3} \theta}$-bimodules

$$
E_{g_{2}^{-1}}\left(g_{2} g_{3} \theta\right) \otimes_{A_{g_{2} g_{3} \theta}} E_{g_{2}}\left(g_{3} \theta\right) \rightarrow A_{g_{3} \theta} .
$$


Since $t_{g_{2}^{-1}, g_{2}}$ induces an isomorphism of the bimodule on the left with $A_{g_{3} \theta}$ we see that this morphism differs from $t_{g_{2}^{-1}, g_{2}}$ by the multiplication with a central element in $A_{g_{3}} \theta$. Since we assumed that $\theta$ is irrational, this central element should be a constant. Thus, it suffices to prove the following identity:

$$
t_{g_{2}^{-1}, g_{2} g_{3}}\left(f_{1} \otimes t_{g_{2}, g_{3}}\left(f_{2} \otimes f_{3}\right)(0,0)=\left(t_{g_{2}^{-1}, g_{2}}\left(f_{1} \otimes f_{2}\right) f_{3}\right)(0,0) .\right.
$$

Also, we can assume that $f_{1}(x, \alpha)=0$ for $\alpha \not \equiv 0\left(c_{1}\right)$ and denote $f_{1}(x)=f_{1}(x, 0)$. Then the LHS of (1.6) can be rewritten as

$$
\begin{aligned}
& \sum_{m, n \in \mathbb{Z}} f_{1}\left(\frac{m}{\operatorname{rk}\left(g_{2}, g_{3} \theta\right)}\right) f_{2}\left(m+\frac{n \operatorname{rk}\left(g_{2}, g_{3} \theta\right)}{c_{2}}, n\right) f_{3}\left(-\frac{n}{c_{3}},-a_{3} n\right)= \\
& \sum_{n \in \mathbb{Z}}\left(U_{1}^{n} f_{3}\right)(0,0) \cdot \sum_{m \in \mathbb{Z}} f_{1}\left(\frac{m}{\operatorname{rk}\left(g_{2}, g_{3} \theta\right)}\right)\left(f_{2} U_{1}^{-n}\right)(m, 0) .
\end{aligned}
$$

Now we can rewrite the inner sum using the Poisson summation formula:

$$
\begin{aligned}
& \sum_{m \in \mathbb{Z}} f_{1}\left(\frac{m}{\operatorname{rk}\left(g_{2}, g_{3} \theta\right)}\right)\left(f_{2} U_{1}^{-n}\right)(m, 0)=\sum_{m \in \mathbb{Z}} \int \exp (2 \pi i m y) f_{1}\left(\frac{y}{\operatorname{rk}\left(g_{2}, g_{3} \theta\right)}\right)\left(f_{2} U_{1}^{-n}\right)(y, 0) d y= \\
& \sum_{m \in \mathbb{Z}} \int f_{1}\left(\frac{y}{\operatorname{rk}\left(g_{2}, g_{3} \theta\right)}\right)\left(f_{2} U_{1}^{-n} U_{2}^{m}\right)(y, 0) d y=\sum_{m \in \mathbb{Z}} b\left(f_{1}, f_{2} U_{1}^{-n} U_{2}^{-m}\right) .
\end{aligned}
$$

Therefore, the LHS of (1.6) is equal to

$$
\sum_{m, n \in \mathbb{Z}}\left(U_{1}^{n} f_{3}\right)(0,0) b\left(U_{1}^{-n} U_{2}^{-m} f_{1}, f_{2}\right)=\sum_{m, n \in \mathbb{Z}}\left(U_{2}^{m} U_{1}^{n} f_{3}\right)(0,0) b\left(U_{1}^{-n} U_{2}^{-m} f_{1}, f_{2}\right)
$$

which is the definition of the RHS of (1.6).

Part (c) follows from the well-known fact that all basic modules considered as bimodules are invertible and from the isomorphism (1.5).

Remark. The cocycle condition 1.1 implies that the pairing $t_{g_{1}, g_{2}}$ is even (where we define the $\mathbb{Z} / 2 \mathbb{Z}$-grading of the tensor product in the usual way).

Corollary 1.4. (a) For every pair of basic modules $E_{g}(\theta), E_{h}(\theta)$ there is a canonical isomorphism

$$
\operatorname{Hom}_{A_{\theta}}\left(E_{g}(\theta), E_{h}(\theta)\right) \simeq E_{h g^{-1}}(g \theta)
$$

of $A_{h \theta}-A_{g \theta}$-bimodules.

(b) For a triple of basic modules $\left(E_{g}(\theta), E_{h}(\theta), E_{k}(\theta)\right)$ the canonical composition map

$$
\operatorname{Hom}_{A_{\theta}}\left(E_{h}(\theta), E_{k}(\theta)\right) \otimes \operatorname{Hom}_{A_{\theta}}\left(E_{g}(\theta), E_{h}(\theta)\right) \rightarrow \operatorname{Hom}_{A_{\theta}}\left(E_{g}(\theta), E_{k}(\theta)\right)
$$

is identified via isomorphisms (1.7) with the pairing $t_{k h^{-1}, h g^{-1}}$.

Remark. Recall that the Morita equivalence associated with a basic module $E_{g_{0}}(\theta)$ is the functor $M \mapsto M \otimes_{A_{g_{0} \theta}} E_{g_{0}}(\theta)$ from the category of right $A_{g_{0} \theta}$-modules to that of right $A_{\theta}$-modules. According to (1.5) it sends $E_{g}\left(g_{0} \theta\right)$ to $E_{g g_{0}}(\theta)$. The corresponding isomorphism

$$
\operatorname{Hom}_{A_{g_{0} \theta}}\left(E_{g}\left(g_{0} \theta\right), E_{h}\left(g_{0} \theta\right)\right) \simeq \operatorname{Hom}_{A_{\theta}}\left(E_{g g_{0}}(\theta), E_{h g_{0}}(\theta)\right)
$$


is compatible with the identification of both sides with $E_{h g^{-1}}\left(g g_{0} \theta\right)$ given in the above corollary.

\section{HolomorPhic STRUCTURES}

2.1. Standard holomorphic structures on basic modules. Let us fix a complex number $\tau$ such that $\operatorname{Im}(\tau) \neq 0$. We will think about $\tau$ as a complex structure on the noncommutative torus $T_{\theta}$. Namely, $\tau$ defines a one-dimensional subalgebra in the Lie algebra of derivations of the algebra $A_{\theta}$ spanned by the derivation $\delta_{\tau}$ given by

$$
\delta_{\tau}\left(\sum_{\left(n_{1}, n_{2}\right) \in \mathbb{Z}^{2}} a_{n_{1}, n_{2}} U_{1}^{n_{1}} U_{2}^{n_{2}}\right)=2 \pi i \sum_{\left(n_{1}, n_{2}\right) \in \mathbb{Z}^{2}}\left(n_{1} \tau+n_{2}\right) a_{n_{1}, n_{2}} U_{1}^{n_{1}} U_{2}^{n_{2}} .
$$

We denote by $T_{\theta, \tau}$ the noncommutative torus $T_{\theta}$ equipped with this complex structure.

Definition. (i) A holomorphic structure on a right $A_{\theta}$-module $E$ (compatible with the complex structure on $\left.T_{\theta, \tau}\right)$ is an operator $\bar{\nabla}: E \rightarrow E$ satisfying the following Leibnitz identity:

$$
\bar{\nabla}(e a)=\bar{\nabla}(a) \cdot a+e \cdot \delta_{\tau}(a),
$$

where $e \in E, a \in A_{\theta}$.

(ii) If $E$ and $E^{\prime}$ are right $A_{\theta}$-modules equipped with holomorphic structures, then we say that a morphism $f: E \rightarrow E^{\prime}$ of right $A_{\theta}$-modules is holomorphic if $\bar{\nabla}(f e)=$ $f(\bar{\nabla} e)$.

(iii) For a right $A_{\theta}$-module $E$ equipped with a holomorphic structure $\bar{\nabla}$ we define the cohomology as follows:

$$
\begin{gathered}
H^{*}(E)=H^{*}(E, \bar{\nabla}):=H^{0}(E, \bar{\nabla}) \oplus H^{1}(E, \bar{\nabla}), \text { where } \\
H^{0}(E, \bar{\nabla}):=\operatorname{ker}(\bar{\nabla}: E \rightarrow E), H^{1}(E, \bar{\nabla}):=\operatorname{coker}(\bar{\nabla}: E \rightarrow E) .
\end{gathered}
$$

It is natural to call projective right $A_{\theta}$-modules equipped with holomorphic structures holomorphic bundles on the complex noncommutative torus $T_{\theta, \tau}$.

We are going to introduce a family of holomorphic structures (depending on one complex parameter) on every basic module. Namely, for $E=E_{n, m}(\theta)$ with $m \neq 0$ we set

$$
\bar{\nabla}_{z}(f)=\frac{\partial f}{\partial x}+2 \pi i(\tau \mu(E) x+z) f
$$

where $\mu(E)=m /(n+m \theta), f \in E, z \in \mathbb{C}$. It is easy to see that for every $z$ this is a holomorphic structure on $E$. For $E=A_{\theta}$ we define holomorphic structures on the trivial module $\bar{\nabla}_{z}: A_{\theta} \rightarrow A_{\theta}$ by setting

$$
\bar{\nabla}_{z}(a)=2 \pi i z \cdot a+\delta_{\tau}(a) .
$$

We will denote by $E_{g}^{z}(\theta)$ the basic module $E_{g}$ equipped with the holomorphic structure $\bar{\nabla}_{z}$.

Definition. A standard holomorphic structure on a basic module $E_{g}$ is one of the structures $\bar{\nabla}_{z}$. A standard holomorphic bundle on $T_{\theta}$ is one of the bundles $E_{g}^{z}(\theta)$. 
Note that there is a natural notion of equivalence of holomorphic structures on the same module $E$ : two such structures $\bar{\nabla}$ and $\bar{\nabla}^{\prime}$ are equivalent if there exists a holomorphic isomorphism $(E, \bar{\nabla}) \rightarrow\left(E, \bar{\nabla}^{\prime}\right)$. In other words, the group of automorphisms of $E$ as a right $A_{\theta}$-module acts on the space of holomorphic structures on $E$, so that equivalence classes are orbits under this action.

Proposition 2.1. (a) For every basic right $A_{\theta}$-module $E=E_{g}(\theta)$ and every $z \in \mathbb{C}$ the operator $\bar{\nabla}_{z}: E \rightarrow E$ satisfies the following Leibnitz rule with respect to the left action of $A_{g \theta}$ on $E$ :

$$
\bar{\nabla}_{z}(b e)=b \cdot \bar{\nabla}_{z}(e)+\frac{1}{\operatorname{rk}(E)} \delta_{\tau}(b) \cdot e,
$$

where $b \in A_{g \theta}, e \in E$.

(b) The holomorphic structures $\bar{\nabla}_{z^{\prime}}$ and $\bar{\nabla}_{z}$ are equivalent if and only if $z^{\prime} \equiv z$ $\bmod \frac{1}{\mathrm{rk}(E)}(\mathbb{Z}+\tau \mathbb{Z})$.

(c) Let $E_{0}=E_{g}(\theta)$ be a basic right $A_{\theta}$-module equipped with a standard holomorphic structure $\bar{\nabla}_{z}, E$ be a right $A_{g \theta}$-module. Then for every holomorphic structure $\bar{\nabla}: E \rightarrow E$ on $E$ the formula

$$
\bar{\nabla}\left(e \otimes e_{0}\right)=\frac{1}{\operatorname{rk}\left(E_{0}\right)} \bar{\nabla}(e) \otimes e_{0}+e \otimes \bar{\nabla}_{z}\left(e_{0}\right)
$$

defines a holomorphic structure on $E \otimes_{A_{g \theta}} E_{0}$.

Proof. (a) This follows from the explicit formulas for the action of $A_{g \theta}$ on $E$.

(b) The identities of (a) for $b=U_{1}$ and $b=U_{2}$ can be rewritten as follows:

$$
\begin{aligned}
& \bar{\nabla}_{z-\frac{\tau}{\mathrm{rk}(E)}}\left(U_{1} e\right)=U_{1} \bar{\nabla}_{z}(e), \\
& \bar{\nabla}_{z-\frac{1}{\mathrm{rk}(E)}}\left(U_{2} e\right)=U_{2} \bar{\nabla}_{z}(e) .
\end{aligned}
$$

Therefore, $U_{1}$ (resp. $U_{2}$ ) induces an equivalence between $\bar{\nabla}_{z}$ and $\bar{\nabla}_{z-\frac{\tau}{\mathrm{rk}(E)}}$ (resp. $\left.\bar{\nabla}_{z-\frac{1}{\operatorname{rk}(E)}}\right)$.

Conversely, assume that $\bar{\nabla}_{z^{\prime}}$ is equivalent to $\bar{\nabla}_{z}$. Then $z^{\prime}=z-\delta_{\tau}(u) u^{-1} / \operatorname{rk}(E)$ for some $u \in A_{g \theta}^{*}$. But this implies that $\delta_{\tau}(u)$ is proportional to $u$, which is possible only for $u$ of the form $c \cdot U_{1}^{n_{1}} U_{2}^{n_{2}}$. Hence, constant elements $z$ and $z^{\prime}$ belong to the same orbit if and only if $z-z^{\prime} \in \operatorname{rk}(E)^{-1}(\mathbb{Z}+\tau \mathbb{Z})$.

(c) This follows easily from (a).

It is easy to see that not every holomorphic structure on a basic module is equivalent to a standard one. Indeed, if $\theta$ is irrational then every basic module $E$ is isomorphic to a direct sum $E_{1} \oplus E_{2} \oplus \ldots E_{n}$ of two or more other basic modules (this follows from Rieffel's cancellation theorem, see [14]). Then the direct sum of holomorphic structures on $E_{i}$ 's will not be equivalent to a standard holomorphic structure on $E$, since it will admit holomorphic endomorphisms that are not proportional to the identity, while for standard holomorphic structures this is not the case(as follows from Corollary 2.3 below). Holomorphic bundles isomorphic to standard ones can be characterized as those bundles admitting a compatible unitary connection with constant curvature (and such that the underlying projective module is basic). In the commutative case the theorem of Narasimhan and Seshadri [11] asserts that these are exactly stable bundles. In case of elliptic curves 
an equivalent characterization is that these are bundles with only scalar endomorphisms. One may hope that this remains true for noncommutative tori. One natural approach to this problem would be to mimick the method of Donaldson in [4]. Roughly speaking the idea is to apply the gradient flow of the Yang-Mills functional and then use the theorem of Connes-Rieffel [2] stating that the minima of this functional are exactly connections of constant curvature (this approach is advocated in [19]). However, the induction in rank employed in [4] cannot be used for irrational $\theta$, since ranks of bundles on $T_{\theta}$ can be arbitrarily small. It is possible that one can modify the argument using Rieffel's description of all critical sets of the Yang-Mills functional (see [15]). Another possible approach would be to use an analogue of the Fourier-Mukai transform defined in section 3.3.

2.2. Tensor products and Hom in holomorphic category. Now we are going to study the relation between the pairings $t_{g_{1}, g_{2}}$ and holomorphic structures.

Proposition 2.2. (a) For every $g_{1}, g_{2} \in \mathrm{SL}_{2}(\mathbb{Z}), z_{1}, z_{2} \in \mathbb{C}$ one has

$\bar{\nabla}_{z_{1}+z_{2}}\left(t_{g_{1}, g_{2}}\left(f_{1} \otimes f_{2}\right)\right)=t_{g_{1}, g_{2}}\left(\frac{1}{\operatorname{rk}\left(g_{2}, \theta\right)} \bar{\nabla}_{\mathrm{rk}\left(g_{2}, \theta\right) z_{1}}\left(f_{1}\right) \otimes f_{2}\right)+t_{g_{1}, g_{2}}\left(f_{1} \otimes \bar{\nabla}_{z_{2}}\left(f_{2}\right)\right)$,

where $f_{1} \in E_{g_{1}}\left(g_{2} \theta\right), f_{2} \in E_{g_{2}}(\theta)$.

(b) The pairing $t_{g_{1}, g_{2}}$ induces a well-defined pairing

$$
H^{i}\left(E_{g_{1}}\left(g_{2} \theta\right), \bar{\nabla}_{\mathrm{rk}\left(g_{2}, \theta\right) z_{1}}\right) \otimes H^{j}\left(E_{g_{2}}(\theta), \bar{\nabla}_{z_{2}}\right) \rightarrow H^{i+j}\left(E_{g_{1} g_{2}}(\theta), \bar{\nabla}_{z_{1}+z_{2}}\right)
$$

where $(i, j)$ is either $(0,0),(1,0)$ or $(0,1)$.

Proof. (a) Since $\bar{\nabla}_{z}(f)=\bar{\nabla}_{0}(f)+z f$, it suffices to consider the case $z_{1}=z_{2}=0$. If $g_{1} \neq 1, g_{2} \neq 1$ and $g_{1} g_{2} \neq 1$ then (2.2) follows immediately from the explicit formula for the pairing $t_{g_{1}, g_{2}}$. For $g_{2}=1$ it reduces to Leibnitz identity, while for $g_{1}=1$ it follows from Lemma 2.1. Finally, in the case $g_{1} g_{2}=1$ the equality (2.2) follows from the identity

$$
b\left(\frac{1}{\operatorname{rk}(g, \theta)} \bar{\nabla}_{0}\left(f_{1}\right) \otimes f_{2}\right)+b\left(f_{1} \otimes \bar{\nabla}_{0}\left(f_{2}\right)\right)=0
$$

for the pairing (1.3), which is easy to check using integration by parts.

(b) This follows immediately from (a).

Corollary 2.3. Let $E=E_{g}^{z}(\theta)$ and $E^{\prime}=E_{g^{\prime}}^{z^{\prime}}(\theta)$ be a pair of basic modules equipped with holomorphic structures $\bar{\nabla}_{z}$ and $\bar{\nabla}_{z^{\prime}}$ respectively.

(a) The formula

$$
\bar{\nabla}(\phi)(e)=\operatorname{rk}(E)\left[\bar{\nabla}_{z^{\prime}}(\phi(e))-\phi\left(\bar{\nabla}_{z} e\right)\right]
$$

defines a holomorphic structure on the right $A_{g \theta}$-module $\operatorname{Hom}_{A_{\theta}}\left(E, E^{\prime}\right)$ (where $\phi \in$ $\left.\operatorname{Hom}_{A_{\theta}}\left(E, E^{\prime}\right), e \in E\right)$.

(b) Under the isomorphism (1.7) the above holomorphic structure on $\operatorname{Hom}_{A_{\theta}}\left(E, E^{\prime}\right)$ corresponds to the operator $\bar{\nabla}_{\operatorname{rk}(E)\left(z^{\prime}-z\right)}$ on $E_{g^{\prime} g^{-1}}(g \theta)$, i.e. we have

$$
\operatorname{Hom}_{A_{\theta}}\left(E_{g}^{z}(\theta), E_{g^{\prime}}^{z^{\prime}}(\theta)\right) \simeq E_{g^{\prime} g^{-1}}^{\mathrm{rk}(g, \theta)\left(z^{\prime}-z\right)}(g \theta)
$$

in a way compatible with holomorphic structures.

(c) The subspace $H^{0}\left(\operatorname{Hom}_{A_{\theta}}\left(E, E^{\prime}\right)\right) \subset \operatorname{Hom}_{A_{\theta}}\left(E, E^{\prime}\right)$ coincides with the subspace of holomorphic morphisms $E \rightarrow E^{\prime}$. 
In the same way as in commutative geometry we can interpret the space $H^{1}\left(\operatorname{Hom}_{A_{\theta}}\left(E, E^{\prime}\right)\right)$ in terms of holomorphic extensions.

Proposition 2.4. Let $E=E_{g}^{z}(\theta)$ and $E^{\prime}=E_{g^{\prime}}^{z^{\prime}}(\theta)$ be a pair of basic modules equipped with standard holomorphic structures. Then there is a functorial bijection between the space $H^{1}\left(\operatorname{Hom}_{A_{\theta}}\left(E^{\prime}, E\right)\right)$ and isomorphism classes of extensions $0 \rightarrow$ $E \rightarrow F \rightarrow E^{\prime} \rightarrow 0$ in the category of holomorphic bundles on $T_{\theta}$.

Proof. Let $0 \rightarrow E \rightarrow F \rightarrow E^{\prime} \rightarrow 0$ be an extension in the holomorphic category. Since $E^{\prime}$ is projective we can choose a (non-holomorphic) splitting $s: E^{\prime} \rightarrow F$ which induces an isomorphism of $A_{\theta}$-modules $F \simeq E \oplus E^{\prime}$. The holomorphic structure $\bar{\nabla}_{F}$ on $F$ should have form $\left(\begin{array}{cc}\bar{\nabla}_{E} & \phi \\ 0 & \bar{\nabla}_{E^{\prime}}\end{array}\right)$ for some $\phi \in \operatorname{Hom}_{A_{\theta}}\left(E^{\prime}, E\right)$. All possible splittings $s: E^{\prime} \rightarrow F$ form a principal homogeneous space over $\operatorname{Hom}_{A_{\theta}}\left(E^{\prime}, E\right)$ and changing of $s$ by an element $f \in \operatorname{Hom}_{A_{\theta}}\left(E^{\prime}, E\right)$ leads to the change $\phi \mapsto \phi+\bar{\nabla}(f)$, where $\bar{\nabla}$ is the holomorphic structure on $\operatorname{Hom}_{A_{\theta}}\left(E^{\prime}, E\right)$ defined above. This easily implies the assertion.

2.3. Holomorphic vectors and cohomology. The cohomology spaces $H^{*}\left(E_{g}^{z}(\theta)\right):=$ $H^{*}\left(E_{g}(\theta), \bar{\nabla}_{z}\right)$ are easy to compute. Namely, the following result holds.

Proposition 2.5. Assume that $\operatorname{Im}(\tau)<0$. Let $E=E_{g}^{z}(\theta)$ be a basic module equipped with a holomorphic structure $\bar{\nabla}_{z}$.

(a) If $\mu(E)>0$ then $H^{1}(E)=0$ and $H^{0}(E)$ has dimension $\operatorname{deg}(E)$.

(b) If $\mu(E)<0$ then $H^{0}(E)=0$ and $H^{1}\left(E, \bar{\nabla}_{z}\right)$ has dimension $\operatorname{deg}(E)$.

(c) If $z \neq 0$ then $H^{*}\left(A_{\theta}, \bar{\nabla}_{z}\right)=0$.

(d) The spaces $H^{0}\left(A_{\theta}, \bar{\nabla}_{0}\right)$ and $H^{1}\left(A_{\theta}, \bar{\nabla}_{0}\right)$ are 1-dimensional.

Proof. In the case $\operatorname{deg}(E) \neq 0$ the problem reduces to the computation of the cohomology of the operator

$$
f \mapsto f^{\prime}+2 \pi i(\tau \mu(E) x+z) f
$$

on the Schwartz space $\mathcal{S}(\mathbb{R})$. Now one has to use the fact that for every complex number $a$ with $\operatorname{Re}(a) \neq 0$ the operator $f \mapsto f^{\prime}+(a x+z) f$ on $\mathcal{S}(\mathbb{R})$ has 1 -dimensional kernel and no cokernel (resp., 1-dimensional cokernel and no kernel) if $\operatorname{Re}(a)>0$ (resp. $\operatorname{Re}(a)<0)$. To prove this fact one can use conjugation by the operator $f \mapsto f \exp \left(i \operatorname{Im}(a) x^{2} / 2\right)$ and rescaling the variable $x$ to reduce oneself to the case $a= \pm 1$. Furthermore, making the change of the variable $x \mapsto x \pm \operatorname{Re}(z)$ and conjugating by the operator $f \mapsto f \exp (i \operatorname{Im}(z) x)$ we can assume that $z=0$. Now writing $f$ as a linear combination of $\left(H_{n}(x) \exp \left(-x^{2} / 2\right), n \geq 0\right)$, where $H_{n}$ are Hermite polynomials, one easily proves that $\exp \left(-x^{2} / 2\right)$ generates the kernel for $a=1$ (resp., the cokernel for $a=-1$ ). $\bar{\nabla}_{z}$

In the case $E=A_{\theta}$ the assertion follows immediately from the formula defining

Corollary 2.6. Assume that $\operatorname{Im}(\tau)<0$. Then for every basic module E equipped with some holomorphic structure one has

$$
\chi(E)=\operatorname{dim} H^{i}(E)-\operatorname{dim} H^{i+1}(E)=\operatorname{deg}(E),
$$

where $i=0$ if $\operatorname{rk}(E)>0$ and $i=1$ if $\operatorname{rk}(E)<0$. 
The computation of 2.5 gives explicit bases in $H^{*}\left(E_{g}^{z}(\theta)\right)$. In particular, the space of holomorphic vectors $H^{0}\left(E_{g}^{z}(\theta)\right)$ in a basic module $E_{g}^{z}(\theta)$, such that $\operatorname{deg}(g)>0$, has a natural basis

$$
\phi_{\alpha}^{z}(x, \beta):=\mathrm{e}\left(-\tau \mu(E) x^{2} / 2-z x\right) \delta_{\alpha}(\beta), \alpha \in \mathbb{Z} / \operatorname{deg}(g) \mathbb{Z},
$$

where $\mathrm{e}(t):=\exp (2 \pi i t), \delta_{\alpha}$ is the delta-function at $\alpha \in \mathbb{Z} / \operatorname{deg}(g) \mathbb{Z}$. The basis in $H^{0}\left(A_{\theta}, \bar{\nabla}_{0}\right)$ is the element $\phi^{0}=1 \in A_{\theta}$.

The following proposition is essentially equivalent to the main result of [3].

Proposition 2.7. For every $g_{1}, g_{2} \in \mathrm{SL}_{2}(\mathbb{Z})$ such that $\operatorname{deg}\left(g_{1}\right)>0$, $\operatorname{deg}\left(g_{2}\right)>0$, $\operatorname{rk}\left(g_{1}, \theta\right)>0, \operatorname{rk}\left(g_{2}, \theta\right)>0$ one has $\operatorname{deg}\left(g_{1} g_{2}\right)>0$ and

$$
\begin{gathered}
t_{g_{1}, g_{2}}\left(\phi_{\alpha_{1}}^{\mathrm{rk}\left(g_{2}, \theta\right) z_{1}} \otimes \phi_{\alpha_{2}}^{z_{2}}\right)=\sum_{\alpha \in \mathbb{Z} / c_{12} \mathbb{Z}} c_{\alpha_{1}, \alpha_{2}}^{\alpha} \phi_{\alpha}^{z_{1}+z_{2}}, \text { where } \\
c_{\alpha_{1}, \alpha_{2}}^{\alpha}=\sum_{m \in I_{g_{1}, g_{2}}\left(\alpha_{1}, \alpha_{2}, \alpha\right)} \mathrm{e}\left[\frac{-\tau m^{2} / 2+\left(c_{1} z_{2}-\operatorname{rk}\left(g_{1} g_{2}, \theta\right) c_{2} z_{1}\right) m}{c_{1} c_{2} c_{12}}\right],
\end{gathered}
$$

where we use the notation of Proposition 1.2.

Proof. The fact that $\operatorname{deg}\left(g_{1} g_{2}\right)>0$ follows from the identity

$$
\operatorname{deg}\left(g_{1} g_{2}\right)=\operatorname{deg}\left(g_{1}\right) \operatorname{rk}\left(g_{2}^{-1}, \theta\right)+\operatorname{deg}\left(g_{2}\right) \operatorname{rk}\left(g_{1}, \theta\right)
$$

which is the particular case of (1.2). Hence, $g_{1} g_{2} \neq 1$, and we can use the formula of Proposition 1.2(a) to compute $t_{g_{1}, g_{2}}\left(\phi_{\alpha_{1}}^{\mathrm{rk}\left(g_{2}, \theta\right) z_{1}} \otimes \phi_{\alpha_{2}}^{z_{2}}\right)$. This gives the above formula for $c_{\alpha_{1}, \alpha_{2}}^{\alpha}$.

The main thing to observe in the formula of the above proposition is that $\theta$ enters only through the combination $\operatorname{deg}\left(g_{1}\right) z_{2}-\operatorname{rk}\left(g_{1} g_{2}, \theta\right) \operatorname{deg}\left(g_{2}\right) z_{1}$.

2.4. Translations, tensorings, pull-back and push-forward with respect to isogenies. To conclude this section let us consider noncommutative analogues of some natural operations on holomorphic vector bundles. For every $\left(v_{1}, v_{2}\right) \in \mathbb{R}^{2}$ we denote by $\alpha_{v_{1}, v_{2}}$ the automorphism $U_{1} \mapsto \mathrm{e}\left(v_{1}\right) U_{1}, U_{2} \mapsto \mathrm{e}\left(v_{2}\right) U_{2}$ of $A_{\theta}$. Then for a right $A_{\theta}$-module $E$ we denote by $t_{\left(v_{1}, v_{2}\right)}^{*} E$ the space $E$ with the new right action of $A_{\theta}$ given by $f \mapsto f \cdot \alpha_{v_{1}, v_{2}}^{-1}(a)$, where $a \in A_{\theta}$. Since $\delta_{\tau}$ commutes with $A_{v_{1}, v_{2}}$, a holomorphic structure $\bar{\nabla}: E \rightarrow E$ induces a holomorphic structure on $t_{\left(v_{1}, v_{2}\right)}^{*} E$ (given by the same operator).

Another natural operation is an analogue of tensoring with a topologically trivial line bundle. Clearly, analogues of such line bundles are modules $E_{1}^{\lambda}(\theta)$, where $\lambda \in \mathbb{C}$ (recall that $E_{1}^{\lambda}(\theta)$ is $A_{\theta}$ equipped with the holomorphic structure $\bar{\nabla}_{\lambda}=\delta_{\tau}+2 \pi i \lambda$ ). It is easy to see that for every right $A_{\theta}$-module $E$ with holomorphic structure $\bar{\nabla}$ the tensor product $E \otimes_{A_{\theta}} E_{1}^{\lambda}(\theta)$ is isomorphic to $E$ as a $A_{\theta}$-module, but the holomorphic structure on it (defined in Proposition 2.1(c)) corresponds to $\bar{\nabla}+2 \pi i \lambda$ under this isomorphism. Thus, we have

$$
E_{g}^{z}(\theta) \otimes_{A_{\theta}} E_{1}^{\lambda} \simeq E_{g}^{z+\lambda} .
$$

Therefore, using Proposition 2.1(b), we obtain an isomorphism

$$
E \otimes_{A_{\theta}} E_{1}^{\frac{m+n \tau}{\mathrm{rk}(E)}} \simeq E
$$

for $m, n \in \mathbb{Z}$. 
For a standard holomorphic bundle $E=E_{g}^{z}(\theta)$ such that $\operatorname{deg}(E) \neq 0$, the map

$$
f(x) \mapsto \mathrm{e}\left(\mu(E) v_{1} x\right) f\left(x+v_{2}\right)
$$

induces an isomorphism

$$
t_{\left(v_{1}, v_{2}\right)}^{*} E \widetilde{\rightarrow} E \otimes E_{1}^{\mu(E)\left(\tau v_{2}-v_{1}\right)}
$$

compatible with holomorphic structures. On the other hand, we have isomorphisms

$$
t_{\left(v_{1}, v_{2}\right)}^{*} E_{1}^{z} \simeq E_{1}^{z}
$$

for all $\left(v_{1}, v_{2}\right) \in \mathbb{R}^{2}$ and all $z \in \mathbb{C}$ given by the map $\alpha_{v_{1}, v_{2}}: E_{1} \rightarrow E_{1}$.

Note that if $c=\operatorname{deg}(E) \neq 0$ then taking in $(2.3)\left(v_{1}, v_{2}\right)=(m / c, n / c)$ with $m, n \in \mathbb{Z}$ we obtain isomorphisms

$$
t_{(m / c, n / c)}^{*} E \rightarrow E
$$

of holomorphic vector bundles on $T_{\theta, \tau}$. Therefore, combining induced isomorphisms of cohomology spaces with the natural identifications $H^{*}\left(t_{(m / c, n / c)}^{*} E\right)=H^{*}(E)$ we obtain a collection of invertible operators

$$
U_{(m / c, n / c)}: H^{i}(E) \rightarrow H^{i}(E),
$$

where $i$ is the unique degree such that $H^{i}(E) \neq 0$. Similar to the commutative case we have the following result.

Lemma 2.8. The operators $U_{(m / c, n / c)}$ define an action on $H^{i}(E)$ of the Heisenberg group $H$ which is a central extension of $(\mathbb{Z} / c \mathbb{Z})^{2}$ by $U(1)$, such that $U(1)$ acts in the standard way. This representation is irreducible.

Proof. Unravelling the definitions we get the following formula for $U_{(m / c, n / c)}$ (up to a slight rescaling):

$$
U_{(m / c, n / c)} f(x, \alpha)=\mathrm{e}\left(-\frac{m}{c} \alpha\right) f(x, \alpha-n a),
$$

where $a \in \mathbb{Z}$ is relatively prime to $c$. Hence, the action of these operators on $H^{i}(E)$ is equivalent to the standard representation of the Heisenberg group on the space of functions on $\mathbb{Z} / c \mathbb{Z}$.

There are also analogues of pull-back and push-forward with respect to isogenies in our situation. Namely, for every positive integer $N$ we have a noncommutative morphism $\pi: T_{\theta} \rightarrow T_{N \theta}$ corresponding to the embedding of algebras

$$
A_{N \theta} \rightarrow A_{\theta}: U_{1} \mapsto U_{1}^{N}, U_{2} \mapsto U_{2} .
$$

If $\tau \in \mathbb{C} \backslash \mathbb{R}$ then $\pi$ can be considered as a morphism of complex noncommutative tori $T_{\theta, \tau} \rightarrow T_{N \theta, N \tau}$. Indeed, the derivation $\delta_{\tau}$ preserves the subalgebra $A_{N \theta} \subset A_{\theta}$ and $\left.\delta_{\tau}\right|_{A_{N \theta}}=\delta_{N \tau}$. For a projective right $A_{\theta}$-module $E$ we set $\pi_{*}(E)=E$, considered as a $A_{N \theta}$-module. Clearly, a holomorphic structure on $E$ induces a holomorphic structure on $\pi_{*}(E)$ (with respect to $N \tau$ ). The analogue of pull-back is the operation $E \mapsto \pi^{*} E=E \otimes_{A_{N \theta}} A_{\theta}$ on projective $A_{N \theta}$-modules. Here is another description of $\pi^{*} E$ : it is the space of functions $f: \mathbb{Z} \rightarrow E$ satisfying the equation $f(n+N)=$ $f(n) U_{1}^{-1}$ with the action of $A_{\theta}$ given by $f U_{1}(n)=f(n-1), f U_{2}(n)=\mathrm{e}(n \theta) f(n) U_{2}$. A holomorphic structure $\bar{\nabla}: E \rightarrow E$ satisfying the Leibnitz rule with respect to $\delta_{N \tau}$ induces a holomorphic structure on $\pi^{*} E$ by the formula

$$
\bar{\nabla}(f)(n)=\bar{\nabla}(f(n))+2 \pi i n \tau f(n),
$$


where $n \in \mathbb{Z}$. It is easy to check that $\left(\pi^{*}, \pi_{*}\right)$ is an adjoint pair of functors between the categories of holomorphic vector bundles on $T_{N \theta, N \tau}$ and $T_{\theta, \tau}$.

Proposition 2.9. (a) For every $m$ relatively prime with $N$ one has an isomorphism

$$
\pi_{*} E_{1, m}^{z}(\theta) \simeq E_{N, m}^{z}(N \theta)
$$

of holomorphic vector bundles on $T_{N \theta, N \tau}$.

(b) For every $m$ one has an isomorphism

$$
\pi^{*} E_{1, m}^{z}(N \theta) \simeq E_{1, m N}^{z}(\theta)
$$

of holomorphic vector bundles on $T_{\theta, \tau}$.

Proof. (a) The required isomorphism is given by $f(x, \alpha) \mapsto f(x, N \alpha)$.

(b) We can consider elements of $\pi^{*} E_{1, m}^{z}(N \theta)$ as functions $f(x, \alpha, n)$ of $x \in \mathbb{R}, \alpha \in$ $\mathbb{Z} / N \mathbb{Z}$ and $n \in \mathbb{Z}$ satisfying $f(x, \alpha, n+N)=f(x+(1+m N \theta) / m, \alpha+1, n)$. The isomorphism with $E_{1, m N}^{z}(\theta)$ sends such a function to $g(x, n)=f\left(x-\frac{n(1+m N \theta)}{m N}, 0, n\right)$, where $x \in \mathbb{R}$ and $n \in \mathbb{Z} / m N \mathbb{Z}$.

\section{Derived CATEgories of holomorphic Bundles}

3.1. Definition and basic properties. Let us fix a complex number $\tau$ such that $\operatorname{Im}(\tau)<0$. We are going to define certain dg-category $\mathcal{C}(\theta, \tau)$ for the complex noncommutative torus $T_{\theta, \tau}$. The corresponding cohomology category $H^{0}(\mathcal{C}(\theta, \tau))$ can be considered as an analogue of the derived category of holomorphic vector bundles.

Objects of the dg-category $\mathcal{C}=\mathcal{C}(\theta, \tau)$ are of the form $E[n]$, where $E$ is a finitely generated projective right $A_{\theta}$-module such that $\operatorname{rk}(E)>0$, equipped with a complex structure (compatible with $\delta_{\tau}$ ), $n$ is an integer. We will often write $E$ instead of $E[0]$. Morphisms are defined as follows:

$$
\operatorname{Hom}_{\mathcal{C}}^{\bullet}\left(E[n], E^{\prime}\left[n^{\prime}\right]\right)=\left(\operatorname{Hom}_{A_{\theta}}\left(E, E^{\prime}\right) \rightarrow \operatorname{Hom}_{A_{\theta}}\left(E, E^{\prime}\right)\right)\left[n^{\prime}-n\right]
$$

where this two-term complex is placed in degrees $n-n^{\prime}$ and $n-n^{\prime}+1$, the differential sends $f \in \operatorname{Hom}_{A_{\theta}}\left(E, E^{\prime}\right)$ to the $A_{\theta}$-linear map $e \mapsto \bar{\nabla}(\phi(e))-\phi(\bar{\nabla}(x))$. The composition

$$
\operatorname{Hom}_{\mathcal{C}}^{\bullet}\left(E_{2}, E_{3}\right) \otimes \operatorname{Hom}_{\mathcal{C}}^{\bullet}\left(E_{1}, E_{2}\right) \rightarrow \operatorname{Hom}_{\mathcal{C}}^{\bullet}\left(E_{1}, E_{3}\right)
$$

is defined in the obvious way.

We denote by $\mathcal{C}^{s t}=\mathcal{C}^{s t}(\theta, \tau)$ the full subcategory of $\mathcal{C}$ consisting of objects $E[n]$ such that $E$ is a standard holomorphic bundle. Note that in this case the differential on $\operatorname{Hom}_{A_{\theta}}\left(E, E^{\prime}\right)$ is proportional to the holomorphic structure $\bar{\nabla}$ on it defined in Corollary 2.3.

Remark. One can also consider the $\mathbb{Z} / 2 \mathbb{Z}$-graded analogue of $\mathcal{C}$. Recall that the $A_{g \theta}$-module $\operatorname{Hom}_{A_{\theta}}\left(E, E^{\prime}\right)$ can be either even or odd (depending on the sign of its rank). We can equip the space $\operatorname{Hom}_{\mathcal{C}}\left(E, E^{\prime}\right)$ with the induced $\mathbb{Z} / 2 \mathbb{Z}$-grading, so that the differential is odd.

We are going to check that in the case $\theta=0$ the category $H^{0} \mathcal{C}^{s t}$ is equivalent to the full subcategory in the derived category $D^{b}(X)$ of coherent sheaves on the elliptic curve $X=X_{\tau}=\mathbb{C} /(\mathbb{Z}+\tau \mathbb{Z})$. Namely, let us denote by $\mathcal{C}^{s t}(X)$ the dgcategory with objects $E[n]$, where $E$ is a stable holomorphic vector bundle on $X, n \in \mathbb{Z}$. The spaces $\operatorname{Hom}_{\mathcal{C}^{s t}(X)}\left(E_{1}, E_{2}\right)$ are given by the Dolbeault complexes of 
$E_{1}^{\vee} \otimes E_{2}$. Then this definition is extended to objects of the form $E[n]$ as before. The associated usual category $H^{0} \mathcal{C}^{s t}(X)$ is the full subcategory in the derived category $D^{b}(X)$.

Proposition 3.1. The dg-categories $\mathcal{C}^{\text {st }}(0, \tau)$ and $\mathcal{C}^{\text {st }}\left(X_{\tau}\right)$ are equivalent. If $\operatorname{Im}(\tau)<$ 0 then under this equivalence $E_{n, m}^{z}$ corresponds to a stable vector bundle of rank $n$ and degree $m$ on $X_{\tau}$.

Proof. Let us define real coordinates $(x, y)$ on $\mathbb{C}$ by setting $z=x-\tau y$. Then we can identify the algebra of smooth functions on the torus $X=X_{\tau}$ with $A_{0}$ by setting $U_{1}=\exp (2 \pi i x), U_{2}=\exp (2 \pi i y)$. Then the derivation $\delta_{\tau}$ on $A_{0}$ is proportional to $\bar{\partial}$. Given a holomorphic vector bundle $V$ over $X$ the space $C^{\infty}(V)$ of smooth sections of $V$ is a projective $A_{0}$-module. Furthermore, trivializing the bundle of $(0,1)$-forms on $X$ we can consider the operator $\bar{\partial}: C^{\infty}(V) \rightarrow C^{\infty}(V)$ (appropriately rescaled) as a holomorphic structure on the $A_{0}$-module $C^{\infty}(V)$.

Let us check that if $V$ is stable then $C^{\infty}(V)$ is a basic $A_{0}$-module with a standard holomorphic structure. Assume first that $V$ is a line bundle of degree $c \neq 0$. Every such line bundle on $X$ is isomorphic to a line bundle $L_{c}(u)$, where $u \in \mathbb{C}$, such that $C^{\infty}\left(L_{c}(u)\right)$ is the space of smooth functions $f$ on $\mathbb{C}$ satisfying the equations $f(z+1)=f(z), f(z-\tau)=\mathrm{e}(u-c z) f(z)$, and holomorphic structure is given by the $\bar{\partial}$-operator with respect to the complex variable $z$. Using the real coordinates $(x, y)$ such that $z=x-\tau y$ we can write a section of $L_{c}(u)$ in the form

$$
f(z)=\sum_{n \in \mathbb{Z}} f_{n}(y) \mathrm{e}(n x)
$$

where $f_{n}(y+1)=f_{n+c}(y) \mathrm{e}(u+c \tau y)$. Setting

$$
g_{n}(y)=f_{n}(y) \mathrm{e}\left(-c \tau y^{2} / 2+(c \tau / 2-u) y\right),
$$

we note that $g_{n}(y+1)=g_{n+c}(y)$, so the function $\phi(y, \alpha)=g_{\alpha}(y-\alpha / c)$ depends only on $\alpha \bmod c \mathbb{Z}$. Smoothness of $f$ implies that the functions $\phi(y, \alpha)$ belong to the Schwartz spaces. It is easy to check that the map $f \mapsto \phi(y, \alpha)$ induces an isomorphism of $A_{0}$-modules $C^{\infty}\left(L_{c}(u)\right) \simeq E_{1, c}(0)$. Furthermore, the holomorphic structure on $L_{c}(u)$ corresponds to the holomorphic structure $\bar{\nabla}_{u-c \tau / 2}$ (with respect to the complex structure $\delta_{\tau}$ on $A_{0}$ ).

Next, we observe that the construction $V \mapsto C^{\infty}(V)$ is compatible with the operation $\pi_{*}$, where $\pi: \mathbb{C} /(d \mathbb{Z}+\tau \mathbb{Z}) \rightarrow \mathbb{C} /(\mathbb{Z}+\tau \mathbb{Z})=X$ is a natural isogeny of degree $d$. Since every stable holomorphic vector bundle of rank $d$ has form $\pi_{*} L_{c}(u)$, applying Proposition 2.9 we derive that the space of smooth sections of such bundle can be identified with a basic module over $A_{0}$ (and that the holomorphic structure on it is standard).

Let us fix a basic right $A_{\theta}$-module $E_{0}=E_{g}(\theta)$ and equip it with a standard holomorphic structure $\bar{\nabla}_{0}$. Then as we have shown in Proposition 2.1, the map $E \mapsto E \otimes_{A_{g \theta}} E_{0}$ extends to a functor from the category of holomorphic bundles over $T_{g \theta}$ to the category of holomorphic bundles over $T_{\theta}$.

Proposition 3.2. The above functor extends to an equivalence of dg-categories $\mathcal{C}(g \theta, \tau) \widetilde{\rightarrow C}(\theta, \tau)$ (resp., $\left.\mathcal{C}^{s t}(g \theta, \tau) \widetilde{\rightarrow} \mathcal{C}^{s t}(\theta, \tau)\right)$. 
Proof. Indeed, for every holomorphic vector bundles $E, E^{\prime}$ over $T_{g \theta}$ we have a natural map

$$
\operatorname{Hom}_{A_{g \theta}}\left(E, E^{\prime}\right) \rightarrow \operatorname{Hom}_{A_{\theta}}\left(E \otimes_{A_{g \theta}} E_{0}, E^{\prime} \otimes_{A_{g \theta}} E_{0}\right)
$$

commuting with the differentials. The inverse $A_{\theta}-A_{g \theta}$-bimodule to $E_{0}$ will define an inverse map.

The following result is an analogue of Serre duality for the category $H^{*}\left(\mathcal{C}^{s t}(\theta, \tau)\right)$.

Proposition 3.3. (a) The pairing (1.3) induces a perfect pairing

$$
H^{i}\left(E_{g^{-1}}^{-\operatorname{rk}(g, \theta) z}(g \theta)\right) \otimes H^{1-i}\left(E_{g}^{z}(\theta)\right) \rightarrow \mathbb{C}
$$

for $i=0,1$.

(b) There is a canonical functorial isomorphism $H^{i} \operatorname{Hom}_{\mathcal{C}}\left(E, E^{\prime}\right) \simeq H^{1-i} \operatorname{Hom}_{\mathcal{C}}\left(E^{\prime}, E\right)^{*}$, where $E, E^{\prime} \in \mathcal{C}^{s t}$.

Proof. (a) The identity

$$
b\left(\frac{1}{\operatorname{rk}(g, \theta)} \bar{\nabla}_{-\operatorname{rk}(g, \theta) z}\left(f_{1}\right) \otimes f_{2}\right)+b\left(f_{1} \otimes \bar{\nabla}_{z}\left(f_{2}\right)\right)=0,
$$

where $f_{1} \in E_{g^{-1}}(g \theta), f_{2} \in E_{g}(\theta)$, ensures that $b$ descends to the required pairing. We already know that dimensions of both spaces are the same. To prove that this pairing is non-degenerate it suffices to check non-degeneracy of the pairing

$$
\operatorname{ker}(d / d x+x: \mathcal{S}(\mathbb{R}) \rightarrow \mathcal{S}(\mathbb{R})) \otimes \operatorname{coker}(d / d x-x: \mathcal{S}(\mathbb{R}) \rightarrow \mathcal{S}(\mathbb{R})) \rightarrow \mathbb{C}
$$

induced by $\left(f_{1}, f_{2}\right)=\int_{x \in \mathbb{R}} f_{1}(x) f_{2}(x) d x$. But both spaces are generated by $\exp \left(-x^{2} / 2\right)$ and $\left(\exp \left(-x^{2} / 2\right), \exp \left(-x^{2} / 2\right)\right) \neq 0$.

(b) This follows from (a) and from the isomorphisms of Corollary 2.3(b).

3.2. Comparison of the categories for different $\theta$. Proposition 2.7 gives explicit formulas for the structure constants of the composition law in the categories $H^{*} \mathcal{C}^{s t}(\theta, \tau)$. Looking at these formulas one can make the following observation. Let us denote by $\mathcal{C}^{s t}(\theta, \tau)_{\theta^{\prime}}$ the full dg-subcategory of $\mathcal{C}^{s t}(\theta, \tau)_{\theta^{\prime}}$ formed by objects $E_{g}^{z}(\theta)[n]$ with $\operatorname{rk}\left(g, \theta^{\prime}\right) \neq 0$.

Theorem 3.4. For every pair $\left(\theta, \theta^{\prime}\right)$ the graded categories $H^{*} \mathcal{C}^{s t}(\theta, \tau)_{\theta^{\prime}}$ and $H^{*} \mathcal{C}^{s t}\left(\theta^{\prime}, \tau\right)_{\theta}$ are equivalent.

Proof. The main ingredient of the proof is the following computation of the composition in the cohomology category of $\mathcal{C}^{s t}(\theta)=\mathcal{C}^{s t}(\theta, \tau)$. Assume that $\operatorname{deg}\left(g_{2} g_{1}^{-1}\right)>0$, $\operatorname{deg}\left(g_{3} g_{2}^{-1}\right)>0$, and $\operatorname{rk}\left(g_{i}, \theta\right)>0$ for $i=1,2,3$. Then the composition

$$
H^{0} \operatorname{Hom}\left(E_{g_{2}}^{z_{2}}(\theta), E_{g_{3}}^{z_{3}}(\theta)\right) \otimes H^{0} \operatorname{Hom}\left(E_{g_{1}}^{z_{1}}(\theta), E_{g_{2}}^{z_{2}}(\theta)\right) \rightarrow H^{0} \operatorname{Hom}\left(E_{g_{1}}^{z_{1}}(\theta), E_{g_{3}}^{z_{3}}(\theta)\right)
$$

can be identified using (1.7) and Corollary 2.3 with the map

$$
H^{0}\left(E_{g_{3} g_{2}^{-1}}^{\mathrm{rk}\left(g_{2}, \theta\right)\left(z_{3}-z_{2}\right)}\left(g_{2} \theta\right)\right) \otimes H^{0}\left(E_{g_{2} g_{1}^{-1}}^{\mathrm{rk}\left(g_{1}, \theta\right)\left(z_{2}-z_{1}\right)}\left(g_{1} \theta\right)\right) \rightarrow H^{0}\left(E_{g_{3} g_{1}^{-1}}^{\mathrm{rk}\left(g_{1}, \theta\right)\left(z_{3}-z_{1}\right)}\left(g_{1} \theta\right)\right)
$$

induced by the pairing $t_{g_{3} g_{2}^{-1}, g_{2} g_{1}^{-1}}$. Applying Proposition 2.7 we get

$$
t_{g_{3} g_{2}^{-1}, g_{2} g_{1}^{-1}}\left(\phi_{\alpha}^{\mathrm{rk}\left(g_{2}, \theta\right)\left(z_{3}-z_{2}\right)} \otimes \phi_{\beta}^{\mathrm{rk}\left(g_{1}, \theta\right)\left(z_{2}-z_{1}\right)}\right)=\sum_{\gamma} c_{\alpha, \beta}^{\gamma} \phi_{\gamma}^{\mathrm{rk}\left(g_{1}, \theta\right)\left(z_{3}-z_{1}\right)}, \text { where }
$$




$$
\begin{aligned}
& c_{\alpha, \beta}^{\gamma}=\sum_{m \in I_{g_{3} g_{2}^{-1}, g_{2} g_{1}^{-1}(\alpha, \beta, \gamma)}} \\
& \mathrm{e}\left(\frac{-\tau m^{2} / 2+\left[\operatorname{deg}\left(g_{3} g_{2}^{-1}\right) \operatorname{rk}\left(g_{1}, \theta\right)\left(z_{2}-z_{1}\right)-\operatorname{deg}\left(g_{2} g_{1}^{-1}\right) \operatorname{rk}\left(g_{3}, \theta\right)\left(z_{3}-z_{2}\right)\right] m}{\operatorname{deg}\left(g_{3} g_{2}^{-1}\right) \operatorname{deg}\left(g_{2} g_{1}^{-1}\right) \operatorname{deg}\left(g_{3} g_{1}^{-1}\right)}\right) .
\end{aligned}
$$

Therefore, $c_{\alpha, \beta}^{\gamma}$ depends on $\theta$ only through the expression

$$
\operatorname{deg}\left(g_{3} g_{2}^{-1}\right) \operatorname{rk}\left(g_{1}, \theta\right)\left(z_{1}-z_{2}\right)+\operatorname{deg}\left(g_{2} g_{1}^{-1}\right) \operatorname{rk}\left(g_{3}, \theta\right)\left(z_{3}-z_{2}\right) .
$$

Applying identity (1.2) we can rewrite this as

$$
\operatorname{deg}\left(g_{3} g_{2}^{-1}\right) \operatorname{rk}\left(g_{1}, \theta\right) z_{1}-\operatorname{deg}\left(g_{3} g_{1}^{-1}\right) \operatorname{rk}\left(g_{2}, \theta\right) z_{2}+\operatorname{deg}\left(g_{2} g_{1}^{-1}\right) \operatorname{rk}\left(g_{3}, \theta\right) z_{3},
$$

so we see that this expression for data $\left(z_{1}, z_{2}, z_{3}, \theta\right)$ is equal to a similar expression for $\left(z_{1}^{\prime}, z_{2}^{\prime}, z_{3}^{\prime}, \theta^{\prime}\right)$, where $z_{i}^{\prime}=z_{i} \operatorname{rk}\left(g_{i}, \theta\right) / \operatorname{rk}\left(g_{i}, \theta^{\prime}\right)$.

Now we can construct the equivalence functor $F=F_{\theta, \theta^{\prime}}: H^{*}\left(\mathcal{C}^{s t}(\theta)_{\theta^{\prime}}\right) \rightarrow$ $H^{*}\left(\mathcal{C}^{s t}\left(\theta^{\prime}\right)_{\theta}\right)$. Without loss of generality we can assume that $\theta<\theta^{\prime}$. For every $g \in \mathrm{SL}_{2}(\mathbb{Z})$ let us denote $\lambda(g)=\operatorname{rk}(g, \theta) / \operatorname{rk}\left(g, \theta^{\prime}\right)$. Then $F$ is defined on objects by setting

$$
F\left(E_{g}^{z}(\theta)[n]\right)= \begin{cases}E_{g}^{\lambda(g) z}\left(\theta^{\prime}\right)[n], & \operatorname{rk}\left(g, \theta^{\prime}\right)>0, \\ E_{-g}^{\lambda(g) z}\left(\theta^{\prime}\right)[n-1], & \operatorname{rk}\left(g, \theta^{\prime}\right)<0 .\end{cases}
$$

We have to define isomorphisms

$F_{g_{1}, g_{2}}: H^{i} \operatorname{Hom}_{\mathcal{C}(\theta)}\left(E_{g_{1}}^{z_{1}}(\theta), E_{g_{2}}^{z_{2}}(\theta)\right) \rightarrow H^{i+\epsilon_{1}-\epsilon_{2}} \operatorname{Hom}_{\mathcal{C}\left(\theta^{\prime}\right)}\left(E_{(-1)^{\epsilon_{1}} g_{1}}^{\lambda\left(g_{1}\right) z_{1}}\left(\theta^{\prime}\right), E_{(-1)^{\epsilon_{2}} g_{2}}^{\lambda\left(g_{2}\right) z_{2}}\left(\theta^{\prime}\right)\right)$ compatible with the composition, where $\epsilon_{i} \in\{0,1\}$ are defined by $(-1)^{\epsilon_{i}}=\operatorname{sign} \operatorname{rk}\left(g_{i}, \theta^{\prime}\right)$. Assume first that $\operatorname{deg}\left(g_{2} g_{1}^{-1}\right)>0$. Then we claim that only the following three cases can occur: (i) $\operatorname{rk}\left(g_{1}, \theta^{\prime}\right)>0, \operatorname{rk}\left(g_{2}, \theta^{\prime}\right)>0$; (ii) $\operatorname{rk}\left(g_{1}, \theta^{\prime}\right)<0, \operatorname{rk}\left(g_{2}, \theta^{\prime}\right)>0$; (iii) $\operatorname{rk}\left(g_{1}, \theta^{\prime}\right)<0, \operatorname{rk}\left(g_{2}, \theta^{\prime}\right)<0$. Indeed, this is clear from the following geometric interpretation of the conditions $\operatorname{rk}(g, \theta)>0, \operatorname{deg}\left(g_{2} g_{1}^{-1}\right)>0$. Consider plane vectors $v_{\theta}=(-\theta, 1)$ and $v_{g}=(d, c)$, where $g=\left(\begin{array}{ll}a & b \\ c & d\end{array}\right)$. Then $\operatorname{rk}(g, \theta)>0$ means that $v_{g}$ belongs to the half-plane $H_{\theta}$ consisting of vectors $v$ such that the pair $\left(v, v_{\theta}\right)$ is positively oriented. The assumption $\theta<\theta^{\prime}$ is equivalent to the condition $-v_{\theta^{\prime}} \in H_{\theta}$. On the other hand, the assumption $\operatorname{deg}\left(g_{2} g_{1}^{-1}\right)>0$ means that the pair $\left(v_{g_{1}}, v_{g_{2}}\right)$ is positively oriented. Since both vectors $v_{g_{1}}$ and $v_{g_{2}}$ belong to the half-plane $H_{\theta}$, our claim follows. In the case (i) we define the map

$$
F_{g_{1}, g_{2}}: H^{0}\left(E_{g_{2} g_{1}^{-1}}^{\mathrm{rk}\left(g_{1}, \theta\right)\left(z_{2}-z_{1}\right)}\left(g_{1} \theta\right)\right) \rightarrow H^{0}\left(E_{g_{2} g_{1}^{-1}}^{\mathrm{rk}\left(g_{1}, \theta^{\prime}\right)\left(\lambda\left(g_{2}\right) z_{2}-\lambda\left(g_{1}\right) z_{1}\right)}\left(g_{1} \theta^{\prime}\right)\right)
$$

by setting

$$
F_{g_{1}, g_{2}}\left(\phi_{\alpha}^{\mathrm{rk}\left(g_{1}, \theta\right)\left(z_{2}-z_{1}\right)}\right)=\phi_{\alpha}^{\mathrm{rk}\left(g_{1}, \theta^{\prime}\right)\left(\lambda\left(g_{2}\right) z_{2}-\lambda\left(g_{1}\right) z_{1}\right)}
$$

for all $\alpha \in \mathbb{Z} / \operatorname{deg}\left(g_{2} g_{1}^{-1}\right) \mathbb{Z}$. In the case (ii) we define the map

$$
F_{g_{1}, g_{2}}: H^{0}\left(E_{g_{2} g_{1}^{-1}}^{\mathrm{rk}\left(g_{1}, \theta\right)\left(z_{2}-z_{1}\right)}\left(g_{1} \theta\right)\right) \rightarrow H^{1}\left(E_{-g_{2} g_{1}^{-1}}^{-\mathrm{rk}\left(g_{1}, \theta^{\prime}\right)\left(\lambda\left(g_{2}\right) z_{2}-\lambda\left(g_{1}\right) z_{1}\right)}\left(-g_{1} \theta^{\prime}\right)\right)
$$

by the formula

$$
F_{g_{1}, g_{2}}\left(\phi_{\alpha}^{\mathrm{rk}\left(g_{1}, \theta\right)\left(z_{2}-z_{1}\right)}\right)=\psi_{-d_{12} \alpha}^{-\operatorname{rk}\left(g_{1}, \theta^{\prime}\right)\left(\lambda\left(g_{2}\right) z_{2}-\lambda\left(g_{1}\right) z_{1}\right)}
$$

where $g_{2} g_{1}^{-1}=\left(\begin{array}{ll}a_{12} & b_{12} \\ c_{12} & d_{12}\end{array}\right),\left(\psi_{\alpha}^{-\operatorname{rk}(g, \theta) z}, \alpha \in \mathbb{Z} / c \mathbb{Z}\right)$ denotes the basis of $H^{1}\left(E_{g^{-1}}^{-\mathrm{rk}(g, \theta) z}(g \theta)\right)$ dual to the basis $\left(\phi_{\alpha}^{z}\right)$ of $H^{0}\left(E_{g}^{z}(\theta)\right)$ with respect to the natural pairing (see 3.3). 
In the case (iii) the definition is similar to the case (i). We can easily extend these definitions to arbitrary morphisms using the compatibility with Serre duality.

It remains to check the compatibility of this functor with the composition

$$
H^{j} \operatorname{Hom}_{\mathcal{C}(\theta)}\left(E_{g_{2}}^{z_{2}}(\theta), E_{g_{3}}^{z_{3}}(\theta)\right) \otimes H^{i} \operatorname{Hom}_{\mathcal{C}(\theta)}\left(E_{g_{1}}^{z_{1}}(\theta), E_{g_{2}}^{z_{2}}(\theta)\right) \rightarrow H^{i+j} \operatorname{Hom}_{\mathcal{C}(\theta)}\left(E_{g_{1}}^{z_{1}}(\theta), E_{g_{3}}^{z_{3}}(\theta)\right)
$$

and the composition of the corresponding objects in $\mathcal{C}^{s t}\left(\theta^{\prime}\right)$. By Serre duality it suffices to consider the case when $\operatorname{deg}\left(g_{2} g_{1}^{-1}\right)>0, \operatorname{deg}\left(g_{3} g_{2}^{-1}\right)>0$ and either $\operatorname{rk}\left(g_{i}, \theta^{\prime}\right)>0$ for $i=1,2,3$, or $\operatorname{rk}\left(g_{1}, \theta^{\prime}\right)<0, \operatorname{rk}\left(g_{2}, \theta^{\prime}\right)>0, \operatorname{rk}\left(g_{3}, \theta^{\prime}\right)>0$. In the former case this compatibility follows from our observation in the beginning of the proof. In the latter case we have to use some cyclic symmetry of the structure constants $c_{\alpha, \beta}^{\gamma}$ appearing above. To reflect the dependence of these constants on various data let us change the notation to $c_{\alpha, \beta}^{\gamma}\left(g_{1}, g_{2}, g_{3} ; z_{1}, z_{2}, z_{3} ; \theta\right)$. The required compatibility follows from the equality

$$
c_{\alpha, \beta}^{\gamma}\left(g_{1}, g_{2}, g_{3} ; z_{1}, z_{2}, z_{3} ; \theta\right)=c_{-d_{13} \gamma, \alpha}^{-d_{12} \beta}\left(g_{2}, g_{3},-g_{1} ; \lambda\left(g_{2}\right) z_{2}, \lambda\left(g_{3}\right) z_{3}, \lambda\left(g_{1}\right) z_{1} ; \theta^{\prime}\right),
$$

where $g_{j} g_{i}^{-1}=\left(\begin{array}{ll}a_{i j} & b_{i j} \\ c_{i j} & d_{i j}\end{array}\right)$. This equality can be easily checked using Lemma $1.3(\mathrm{c})$.

Corollary 3.5. If $\theta$ and $\theta^{\prime}$ are irrational then the graded categories $H^{*}\left(\mathcal{C}^{s t}(\theta, \tau)\right)$ and $H^{*}\left(\mathcal{C}^{s t}\left(\theta^{\prime}, \tau\right)\right)$ are equivalent.

In the case when $\theta$ is irrational and $\theta^{\prime}=0$ we can extend the composition of the above functor $H^{0} \mathcal{C}^{s t}(\theta)_{\theta^{\prime}} \rightarrow H^{0} \mathcal{C}^{s t}(0)$ with the embedding $H^{0} \mathcal{C}^{s t}(0) \subset D^{b}\left(X_{\tau}\right)$ (constructed in Proposition 3.1) to the entire category $H^{0} \mathcal{C}^{s t}(\theta)$. Namely, assuming that $\theta<0$ we extend the functor $F$ above to $E_{g}^{z}(\theta)$ where $g=\left(\begin{array}{cc}0 & 1 \\ -1 & 0\end{array}\right)$ by setting $F\left(E_{g}^{z}(\theta)\right)=\mathcal{O}_{-\theta z}\left(\right.$ this is the structure sheaf of the point $\left.-\theta z \bmod (\mathbb{Z}+\tau \mathbb{Z}) \in X_{\tau}\right)$. The above construction of $F$ on morphisms then can be modified appropriately to prove the equivalence of the category $H^{*}\left(\mathcal{C}^{s t}(\theta, \tau)\right)$ with some full subcategory of the derived category of coherent sheaves on the elliptic curve $\mathbb{C} / \mathbb{Z}+\tau \mathbb{Z}$. Below we will give a more natural construction of the composition of this equivalence with the Fourier-Mukai transform.

3.3. Analogue of the Fourier-Mukai transform. As before we fix a complex structure $\tau$ on $T_{\theta}$ such that $\operatorname{Im}(\tau)<0$. Leibnitz rule implies that for every holomorphic structure $\bar{\nabla}$ on a right $A_{\theta}$-module $E$ the cohomology spaces $H^{*}(E, \bar{\nabla}+2 \pi i z$ id $)$, where $z \in \mathbb{C}$, depend only on $z \bmod (\mathbb{Z}+\tau \mathbb{Z})$. So one can try to define an analogue of the Fourier-Mukai transform of $(E, \bar{\nabla})$ by descending this family of spaces to the elliptic curve $X=X_{\tau}=\mathbb{C} /(\mathbb{Z}+\tau \mathbb{Z})$.

For every $A_{\theta}$-module $E$ let us consider a sheaf of $\mathcal{O}$-modules $E_{\mathbb{C}}$ on $\mathbb{C}$ such that sections of $E_{\mathbb{C}}$ over an open set $U \subset \mathbb{C}$ are $E$-valued holomorphic functions on $U$. We can define two morphisms

$$
\rho_{v}: t_{v}^{*} E_{\mathbb{C}} \underset{\rightarrow}{\rightarrow} E_{\mathbb{C}}
$$

where $v=1$ or $\tau ; t_{v}: \mathbb{C} \rightarrow \mathbb{C}$ denotes the translation $z \mapsto z+v$. Namely, for a local section $f$ of $E_{\mathbb{C}}$ we set

$$
\rho_{v}(f)(z)=f(z+v) U^{v}
$$


where $U^{v}=U_{1}$ or $U_{2}$ for $v=\tau$ or 1 respectively. Clearly, we have

$$
\rho_{1} \circ t_{1}^{*} \rho_{\tau}=\exp (2 \pi i \theta) \rho_{\tau} \circ t_{\tau}^{*} \rho_{1} .
$$

Changing $\rho_{\tau}$ to

$$
\rho_{\tau}^{\prime}=\exp (-2 \pi i \theta z) \rho_{\tau}
$$

we will obtain

$$
\rho_{1} \circ t_{1}^{*} \rho_{\tau}^{\prime}=\rho_{\tau}^{\prime} \circ t_{\tau}^{*} \rho_{1} .
$$

Let us denote by $E_{X}$ the sheaf of $\mathcal{O}$-modules on the elliptic curve $X$ (with respect to the classical topology) obtained from $E_{\mathbb{C}}$ using the $\mathbb{Z}^{2}$-action given by $\rho_{1}$ and $\rho_{\tau}^{\prime}$.

Now assume in addition that $E$ is equipped with a holomorphic structure $\bar{\nabla}$. Then we define $\mathcal{S}(E)=\mathcal{S}(E, \bar{\nabla})$ to be the complex

$$
E_{X} \stackrel{d_{\bar{\nabla}}}{\longrightarrow} E_{X}
$$

of $\mathcal{O}$-modules over $X$, where $d_{\bar{\nabla}}$ is defined in terms of the corresponding operator on $E_{\mathbb{C}}$ :

$$
d_{\bar{\nabla}}(f)(z)=\bar{\nabla}(f(z))+2 \pi i z f(z) .
$$

We leave for the reader to check that this operator is compatible with the $\mathbb{Z}^{2}$-action so it descends to $E_{X}$.

The following basic property is one of the reasons to consider $\mathcal{S}$ as an analogue of the Fourier transform.

Lemma 3.6. For every $\lambda \in \mathbb{C},\left(v_{1}, v_{2}\right) \in \mathbb{R}^{2}$ one has

$$
\begin{gathered}
\mathcal{S}\left(E \otimes E_{1}^{\lambda}\right) \simeq t_{\lambda}^{*} \mathcal{S}(E), \\
\mathcal{S}\left(t_{\left(v_{1}, v_{2}\right)}^{*} E\right) \simeq \mathcal{P}_{\left(v_{1}, v_{2}\right)} \otimes \mathcal{S}(E),
\end{gathered}
$$

where $\mathcal{P}_{\left(v_{1}, v_{2}\right)}$ is the holomorphic bundle on $X$ corresponding to the following action of $\mathbb{Z}+\tau \mathbb{Z}$ on the trivial bundle $\mathcal{O}_{\mathbb{C}}$ :

$$
\rho_{\tau}(f)(z)=\mathrm{e}\left(-v_{1}\right) f(z+\tau), \rho_{1}(f)(z)=\mathrm{e}\left(-v_{2}\right) f(z+1) .
$$

The proof is the direct application of the definitions.

Now let us compute the Fourier transform of standard objects.

Proposition 3.7. (a) Let $E_{n, m}^{0}(\theta)$ be a basic module corresponding to a pair $(n, m)$ such that $m>0$ (and as usual $m \theta+n>0$ ) equipped with the complex structure $\bar{\nabla}_{0}$. Then the complex $\mathcal{S}\left(E_{n, m}^{0}(\theta)\right)$ is quasi-isomorphic to the vector bundle $V_{m,-n}$ on $X$ obtained by the descent from the vector bundle $\mathcal{O}_{\mathbb{C}}^{m}$ with respect to the following action of $\mathbb{Z}+\tau \mathbb{Z}$ :

$$
\rho_{\tau}(f)(z, \alpha)=f(z+\tau, \alpha-1) \mathrm{e}\left(\frac{n}{m} z+\frac{\tau}{2 \mu}\right), \rho_{1}(f)(z, \alpha)=f(z+1, \alpha) \mathrm{e}\left(-\frac{n}{m} \alpha\right),
$$

where we think about sections of $\mathcal{O}_{\mathbb{C}}^{m}$ as functions $f(z, \alpha)$ in $z \in \mathbb{C}$ and $\alpha \in \mathbb{Z} / m \mathbb{Z}$, which are holomorphic in $z$. The vector bundle $V_{m,-n}$ has degree $-n$ and is stable. (b) If $m<0$ (and $m \theta+n>0$ ) then $\mathcal{S}\left(E_{n, m}^{0}(\theta)\right.$ ) is quasi-isomorphic to $V[-1]$, where $V$ is a stable vector bundle on $X$.

(c) The complex $\mathcal{S}\left(E_{1,0}^{z}(\theta)\right)$ is quasi-isomorphic to $\mathcal{O}_{-z}[-1]$, where $\mathcal{O}_{-z}$ is the structure sheaf of the point $-z \bmod (\mathbb{Z}+\tau \mathbb{Z})$ in $X$. 
Proof. (a) Set $E=E_{n, m}^{0}(\theta)$. A version of Proposition 2.5 shows that the morphism $d_{\bar{\nabla}_{0}}: E_{\mathbb{C}} \rightarrow E_{\mathbb{C}}$ is surjective and its kernel is a vector bundle on $\mathbb{C}$. Moreover, we have an explicit isomorphism of the kernel with the rank- $m$ trivial bundle on $\mathbb{C}$ : for a holomorphic function $f(z, \alpha)$ on $U \times \mathbb{Z} / m \mathbb{Z}$, where $U \subset \mathbb{C}$ is an open subset, the function $f(z, \alpha) \mathrm{e}\left(-\tau \mu(E) x^{2} / 2-z x\right)$ defines a section of $\operatorname{ker}\left(d_{\bar{\nabla}_{0}}\right)$ over $U$. It is easy to check that the $\mathbb{Z}^{2}$-action on $\operatorname{ker}\left(d_{\bar{\nabla}_{0}}\right)$ induced by the action of $U_{1}$ and $U_{2}$ coincides with the action defining $V_{m,-n}$. The fact that the vector bundle $V_{m,-n}$ has degree $-n$ and is stable is well-known (note that the sign with $n z / m$ in the definition of $\rho_{\tau}$ is opposite to the standard one due to our assumption $\operatorname{Im}(\tau)<0$ ). (b) The proof of Proposition 2.5 shows that the morphism $d_{\bar{\nabla}_{0}}: E_{\mathbb{C}} \rightarrow E_{\mathbb{C}}$ has no kernel and its cokernel is a bundle $V$ of rank $-m$ on $\mathbb{C}$. Let $g \in \mathrm{SL}_{2}(\mathbb{Z})$ be a matrix with $(m, n)$ as the second row. Proposition 3.3 gives a family of isomorphisms

$$
V_{z}^{*} \simeq H^{0}\left(E_{g^{-1}}^{-\mathrm{rk}(g, \theta) z}(g \theta)\right)
$$

The identifications $V_{z} \simeq V_{z+1}$ and $V_{z} \simeq V_{z+\tau}$ correspond to similar isomorphisms of the $H^{0}$-spaces induced by the left action of generators of $A_{\theta}$ on $E_{g^{-1}}(g \theta)$. In other words, the bundle dual to $V$ (or rather its descent to $X$ ) is isomorphic to the Fourier-Mukai transform of $E_{g^{-1}}^{0}(g \theta)$ considered as a left $A_{\theta}$-module. Therefore, our claim follows from the analogue of part (a) for the Fourier-Mukai transform of left $A_{\theta}$-modules.

(c) The formula for the operator $\bar{\nabla}_{z}: A_{\theta} \rightarrow A_{\theta}$ shows that the complex $\mathcal{S}\left(E_{1,0}^{z_{0}}(\theta)\right)$ is exact outside the point $-z_{0} \bmod (\mathbb{Z}+\tau \mathbb{Z}) \in X$ and that locally near $-z_{0}$ it is quasiisomorphic to the complex $\mathcal{O} \stackrel{z_{0} \dashv z}{\longrightarrow} \mathcal{O}$.

3.4. Equivalences. Let us call an object $E \in D^{b}(X)$ stable if $\operatorname{Hom}(E, E)=\mathbb{C}$. Equivalently, $E \simeq V[n]$ for some $n \in \mathbb{Z}$, where $V$ is either a stable vector bundle or a structure sheaf of a point.

Theorem 3.8. Assume that $\theta$ is irrational. Then the functor $E \mapsto \mathcal{S}(E)$ extends to an equivalence of $H^{0} \mathcal{C}^{s t}(\theta, \tau)$ with the full subcategory of $D^{b}(X)$ consisting of stable objects.

Proof. First of all, we extend $\mathcal{S}$ to all objects of $\mathcal{C}^{s t}(\theta, \tau)$ by requiring that it commutes with the shift $E \mapsto E[1]$. It is clear that a holomorphic map $E \rightarrow E^{\prime}$ induces a map of complexes $\mathcal{S}(E) \rightarrow \mathcal{S}\left(E^{\prime}\right)$. On the other hand, a morphism $E \rightarrow$ $E^{\prime}[1]$ in $H^{0} \mathcal{C}^{s t}(\theta, \tau)$ can be interpreted as an extension class $0 \rightarrow E^{\prime} \rightarrow F \rightarrow E \rightarrow 0$ (see Proposition 2.4). Such an extension induces an exact sequence of complexes $0 \rightarrow \mathcal{S}\left(E^{\prime}\right) \rightarrow \mathcal{S}(F) \rightarrow \mathcal{S}(E) \rightarrow 0$, hence a morphism $\mathcal{S}(E) \rightarrow \mathcal{S}\left(E^{\prime}\right)[1]$ in the derived category. Clearly, these maps define a functor from $H^{0} \mathcal{C}^{s t}(\theta, \tau)$ to $D^{b}(X)$. Using Proposition 3.7 we see that $\mathcal{S}(E)$ is a stable object for every $E \in \mathcal{C}^{s t}(\theta, \tau)$. Let us check that the natural maps

$$
\operatorname{Hom}^{i}\left(E, E^{\prime}\right) \rightarrow \operatorname{Hom}_{D^{b}(X)}^{i}\left(\mathcal{S}(E), \mathcal{S}\left(E^{\prime}\right)\right)
$$

are isomorphisms. The idea of the proof is that both these Hom-spaces are irreducible representations of the same Heisenberg group and that the map between them is a map of Heisenberg modules. Recall that the Heisenberg group action on $\operatorname{Hom}^{i}\left(E, E^{\prime}\right)=H^{i}\left(\operatorname{Hom}_{A_{\theta}}\left(E, E^{\prime}\right)\right)$ was constructed in Lemma 2.8. On the other hand, the Heisenberg group action on $\operatorname{Hom}^{i}(A, B)$ for every pair of stable objects in $D^{b}(X)$ (such that $A \not \subset B[n]$ and $\operatorname{Hom}^{i}(A, B) \neq 0$ ) is constructed as follows. The 
functors of translation by points of $X$ and of tensoring by topologically trivial line bundles on $X$ generate the action of the Heisenberg groupoid $\mathbf{H}$ on $D^{b}(X)$ which is an extension of $X \times X$ by the groupoid of $\mathbb{G}_{m}$-torsors (see [12]). Now for every stable object $A$ in $D^{b}(X)$ let $K_{A} \subset X \times X$ be the subgroup of translations and tensorings preserving $A$ up to an isomorphism. Then the abelian group $K_{A} \cap K_{B}$ is finite and has a natural Heisenberg extension acting on $\operatorname{Hom}^{i}(A, B)$. Indeed, applying an automorphism of $D^{b}(X)$ we can assume that $A=\mathcal{O}_{X}$, in which case this construction is standard (note that $K_{\mathcal{O}_{X}}=X \subset X \times X$ is the subgroup of all translations). The compatibility of $\mathcal{S}$ with Heisenberg group actions on Hom's now follows easily from Proposition 3.6. It remains to check that all the maps (3.1) are non-zero. The commutative diagram

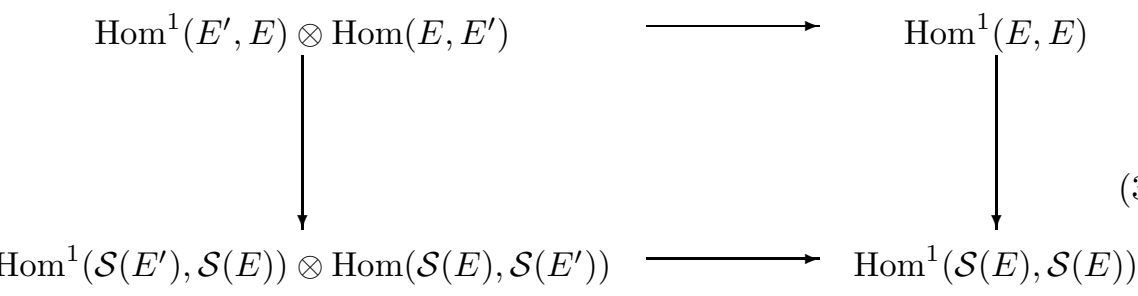

whose rows are perfect pairings, shows that it is enough to prove nonvanishing of the maps

$$
\operatorname{Hom}_{\mathcal{C}^{s t}(\theta, \tau)}^{1}(E, E) \rightarrow \operatorname{Hom}_{D^{b}(X)}^{1}(\mathcal{S}(E), \mathcal{S}(E)) .
$$

This can be done by the direct computation. Let us assume that $E=E_{n, m}^{0}(\theta)$, where $(n, m) \neq(1,0)$ (we leave the case $E=A_{\theta}$ to the reader). Recall that according to Proposition 2.4 a generator of $\operatorname{Hom}^{1}(E, E)$ gives rise to a holomorphic extension

$$
0 \rightarrow E \rightarrow E^{(2)} \rightarrow E \rightarrow 0 .
$$

It is easy to see that $E^{(2)}=E \oplus E$ with the holomorphic structure given by

$$
\left(\begin{array}{cc}
\bar{\nabla}_{0} & 1 \\
0 & \bar{\nabla}_{0}
\end{array}\right) .
$$

Let us assume that $m=\operatorname{deg}(E)>0$ (the case $\operatorname{deg}(E)<0$ is considered similarly). Then $\mathcal{S}(E)$ is the bundle $V_{m,-n}$ described in Proposition 3.7(a). Local sections of $\mathcal{S}\left(E^{(2)}\right)$ over an open subset of $\mathbb{C}$ are described by $E \oplus E$-valued functions of the form

$$
\left(\left(f_{1}(z, \alpha)+f_{2}(z, \alpha) x\right) \mathrm{e}\left(-\tau \mu(E) x^{2} / 2-z x\right), f_{2}(z, \alpha) \mathrm{e}\left(-\tau \mu(E) x^{2} / 2-z x\right)\right),
$$

where $f_{1}(z, \alpha)$ and $f_{2}(z, \alpha)$ are holomorphic in $z$. The $\mathbb{Z}^{2}$-action on pairs $\left(f_{1}(z, \alpha), f_{2}(z, \alpha)\right)$ is given by the rule

$$
\begin{aligned}
& \rho_{\tau}\left(f_{1}, f_{2}\right)=\left(f_{1}(z+\tau, \alpha-1)-\mu f_{2}(z+\tau, \alpha-1), f_{2}(z+\tau, \alpha-1)\right) \mathrm{e}\left(\frac{n}{m} z+\frac{\tau}{2 \mu}\right), \\
& \rho_{1}\left(f_{1}, f_{2}\right)=\left(f_{1}(z+1, \alpha), f_{2}(z+1, \alpha)\right) \mathrm{e}\left(-\frac{n}{m} \alpha\right),
\end{aligned}
$$

where $\mu=\mu(E)$. This easily implies that $\mathcal{S}\left(E^{(2)}\right)$ is a non-split extension of $V_{m,-n}$ by itself.

Remark. It is not difficult to see that the restriction of the functor $\mathcal{S}$ to $H^{0} \mathcal{C}^{s t}(\theta, \tau)_{0}$ is the composition of the functor $F_{\theta, 0}$ from Theorem 3.4 with the embedding of 
$H^{0} \mathcal{C}^{s t}(0, \tau)$ in $D^{b}(X)$ given in Proposition 3.1, followed by the standard FourierMukai transform on $D^{b}(X)$ (where the elliptic curve $X$ is identified with its dual).

Now let us explain the relation between the constructed equivalences and the Morita equivalences. Assume that $\theta<0, \theta^{\prime}=g \theta<0$ and $\operatorname{rk}(g, \theta)>0$. Then the Morita functor from $M_{g, \theta}: \mathcal{C}(g \theta, \tau) \rightarrow \mathcal{C}(\theta, \tau)$ is given by $E \mapsto E \otimes_{A_{g \theta}} E_{g}^{0}(\theta)$. Hence, it maps $E_{h}^{z}\left(\theta^{\prime}\right)$ to $E_{h g}^{u}(\theta)$, where $u=z / \operatorname{rk}(g, \theta)$. On the other hand, the functor $F_{\theta, 0}$ sends an object $E_{h g}^{u}(\theta)$ to $E_{h g}^{u \operatorname{rk}(h g, \theta) / \operatorname{rk}(h g, 0)}(0)$ or $E_{-h g}^{u \operatorname{rk}(h g, \theta) / \operatorname{rk}(h g, 0)}(0)[1]$ depending on whether $\operatorname{rk}(h g, 0)$ is positive or negative. Similarly, $F_{\theta^{\prime}, 0}\left(E_{h}^{z}\left(\theta^{\prime}\right)\right)$ is either $E_{h}^{z \operatorname{rk}\left(h, \theta^{\prime}\right) / \operatorname{rk}(h, 0)}(0)$ or $E_{-h}^{z \operatorname{rk}\left(h, \theta^{\prime}\right) / \operatorname{rk}(h, 0)}(0)[1]$. Note that $u \operatorname{rk}(h g, \theta)=z \operatorname{rk}\left(h, \theta^{\prime}\right)$. It follows that the autoequivalence $G=F_{\theta, 0} \circ M_{g, \theta} \circ F_{\theta^{\prime}, 0}^{-1}$ of the subcategory of stable objects $D^{b}(X)$ sends $E_{h}^{t}(0)$ to $E_{h g}^{t \mathrm{rk}(h, 0) / \mathrm{rk}(h g, 0)}(0)$ or to $E_{-h g}^{t \mathrm{rk}(h, 0) / \mathrm{rk}(h g, 0)}(0)[1]$ depending on the sign of $\operatorname{rk}(h g, 0)$. The autoequivalence $G$ is induced by some autoequivalence of the derived category $D^{b}(X)$ that acts as the matrix $g^{t}$ on the column vector (deg, rk).

3.5. Tilted $t$-structures. Let $\mathcal{C}$ be an abelian category. Recall that a torsion pair in $\mathcal{C}$ is a pair of full subcategories stable under extensions $\left(\mathcal{C}_{1}, \mathcal{C}_{2}\right)$, such that for every $A_{1} \in \mathcal{C}_{1}, A_{2} \in \mathcal{C}_{2}$ one has $\operatorname{Hom}_{\mathcal{C}}\left(A_{1}, A_{2}\right)=0$ and every object $A \in \mathcal{C}$ fits into an exact sequence

$$
0 \rightarrow A_{1} \rightarrow A \rightarrow A_{2} \rightarrow 0
$$

with $A_{1} \in \mathcal{C}_{1}, A_{2} \in \mathcal{C}_{2}$. In this situation the above exact sequence is unique up to a unique isomorphism (hence, it depends functorially on $A$ ).

A torsion pair $p=\left(\mathcal{C}_{1}, \mathcal{C}_{2}\right)$ gives rise to the following $t$-structure in the derived category $D(\mathcal{C})$ :

$$
\begin{aligned}
& D^{p, \leq 0}:=\left\{K \in D(\mathcal{C}): H^{>0}(K)=0, H^{0}(K) \in \mathcal{C}_{1}\right\}, \\
& D^{p, \geq 1}:=\left\{K \in D(\mathcal{C}): H^{<0}(K)=0, H^{0}(K) \in \mathcal{C}_{2}\right\} .
\end{aligned}
$$

The heart of this $t$-structure $\mathcal{C}^{p}:=D^{p, \leq 0} \cap D^{p, \geq 0}$ is equipped with the torsion pair $\left(\mathcal{C}_{2}[1], \mathcal{C}_{1}\right)$.

In the case when $\mathcal{C}$ is the category $\operatorname{Coh}(X)$ of coherent sheaves on an algebraic curve $X$ over a field $k$, there are natural torsion pairs $\left(\operatorname{Coh}_{>\theta}(X), \operatorname{Coh}_{<\theta}(X)\right)$ and $\left(\operatorname{Coh}_{>\theta}(X), \operatorname{Coh}_{<\theta}(X)\right)$ in $\operatorname{Coh}(X)$ associated with every real number $\theta$ (for irrational $\theta$ these two pairs are the same). Namely, for a subset $I \subset \mathbb{R} \cup\{+\infty\}$ we denote by $\operatorname{Coh}_{I}(X)$ the smallest full subcategory in $\operatorname{Coh}(X)$ closed under extensions, containing all simple sheaves with slope $\mu \in I$ (where the slope of a stable bundle is defined as the ratio deg/rk, a slope of a torsion sheaf is defined to be $+\infty)$.

Now let us assume that $\theta$ is irrational and denote by $\left(D^{\theta, \leq 0}, D^{\theta, \geq 1}\right)$ the $t$ structure on $D^{b}(X)$ associated with the torsion pair $\left(\mathrm{Coh}_{>\theta}(X), \mathrm{Coh}_{<\theta}(X)\right)$. Let $\operatorname{Coh}(X)^{\theta}$ be the heart of this $t$-structure, so that $\operatorname{Coh}(X)^{\theta}$ is equipped with the torsion pair $\left(\mathrm{Coh}_{<\theta}[1], \mathrm{Coh}_{>\theta}\right)$.

Proposition 3.9. Assume that $\theta<0$ then the functor $F=F_{\theta, 0}: H^{0} \mathcal{C}^{s t}(\theta) \rightarrow$ $D^{b}(X)$ defined for the pair $(\theta, 0)$ sends the subcategory of holomorphic vector bundles on $T_{\theta}$ to $\operatorname{Coh}(X)^{-\theta^{-1}}[-1]$. 
Proof. Indeed, if $\operatorname{rk}(g, 0)>0$ then $F\left(E_{g}^{z}(\theta)\right)$ is a vector bundle of degree $m$ and rank $n$ such that $m \theta+n>0$, hence $F\left(E_{g}^{z}(\theta)\right) \in \operatorname{Coh}_{<-\theta^{-1}}$. Similarly, if $\operatorname{rk}(g, 0) \leq 0$ then $F\left(E_{g}^{z}(\theta)\right) \in \mathrm{Coh}_{>-\theta^{-1}}[-1]$.

\section{REFERENCES}

[1] A. Connes, $C^{*}$ algèbres et géométrie differentielle, C. R. Acad. Sci. Paris, Sér. A-B, 290 (1980), A599-A604.

[2] A. Connes, M. A. Rieffel, Yang Mills for Non-Commutative Two-Tori, Proceedings of the Conference on Operator Algebras and Mathematical Physics, Univ. of Iowa (1985), 237-266, 1987.

[3] M. Dieng, A. Schwarz, Differential and complex geometry of two-dimensional noncommutative tori, preprint math.QA/0203160.

[4] S. K. Donaldson, A new proof of a theorem of Narasimhan and Seshadri, J. Differential Geom. 18 (1983), 269-277.

[5] M. R. Douglas, Dirichlet branes, homological mirror symmetry, and stability, preprint math.AG/0207021.

[6] K. Fukaya, Floer homology of Lagrangian foliation and noncommutative mirror symmetry I, preprint 1998, http://www.kusm.kyoto-u.ac.jp/ ${ }^{\sim}$ fukaya/fukaya.html

[7] D. Happel, I. Reiten, S. O. Smalo, Tilting in abelian categories and quasitilted algebras, Memoirs AMS 575, 1996.

[8] H. Kajiura, Kronecker foliation, D1-branes and Morita equivalence of Noncommutative twotori, preprint hep-th/0207097.

[9] Yu. I. Manin, Real Multiplication and noncommutative geometry, preprint math.AG/0202109.

[10] S. Mukai, Duality between $D(X)$ and $D(\hat{X})$ with its application to Picard sheaves. Nagoya Math. J. 81 (1981), 153-175.

[11] M. S. Narasimhan, C. S. Seshadri, Stable and unitary vector bundles on a compact Riemann surface, Annals of Math. 82 (1965), 540-567.

[12] A. Polishchuk, Symplectic biextensions and a generalization of the Fourier-Mukai transform, Math. Research Letters 3 (1996), 813-828.

[13] A. Polishchuk, E. Zaslow, Categorical mirror symmetry in the elliptic curve, in Winter School on Mirror Symmetry, Vector Bundles and Lagrangian Submanifolds, 275-295. AMS and International Press, 2001

[14] M. A. Rieffel, The cancellation theorem for projective modules over irrational rotation $\mathbb{C}^{*}$ algebras, Proc. London Math. Soc. (3) 47 (1983), 285-302.

[15] M. A. Rieffel, Critical points of Yang-Mills for noncommutative two-tori, J. Differential Geom. 31 (1990), 535-546.

[16] A. Schwarz, Theta functions on noncommutative tori, Lett. Math. Phys. 58 (2001), 81-90.

[17] Y. Soibelman, Quantum Tori, Mirror symmetry, and Deformation quantization, Lett. Math. Phys. 56 (2001), 99-125.

[18] Y. Soibelman, V. Vologodsky, Non-commutative compactifications and elliptic curves, preprint math.AG/0205117.

[19] M. Spera, Yang Mills theory in non commutative differential geometry, Rendiconti Seminari Facoltà Scienze Univ. Cagliari Supplemento al Vol. 58 (1988), 409-421. 